\title{
Dark matter, neutrino masses and high scale validity of an inert Higgs doublet model
}

\author{
Nabarun Chakrabarty ${ }^{a, 1}$, Dilip Kumar Ghosh ${ }^{b, 2}$, Biswarup Mukhopadhyaya ${ }^{a, 3}$, Ipsita Saha ${ }^{b, 4}$ \\ ${ }^{a}$ Regional Centre for Accelerator-based Particle Physics, Harish-Chandra Research Institute, \\ Chhatnag Road, Jhusi, Allahabad 211019, India \\ ${ }^{b}$ Department of Theoretical Physics, Indian Association for the Cultivation of Science, \\ 2A \& 2B, Raja S.C. Mullick Road, Kolkata 700032, India
}

\begin{abstract}
We consider a two-Higgs doublet scenario containing three $S U(2)_{L}$ singlet heavy neutrinos with Majorana masses. The second scalar doublet as well as the neutrinos are odd under a $Z_{2}$ symmetry. This scenario not only generates Majorana masses for the light neutrinos radiatively but also makes the lighter of the neutral $Z_{2}$-odd scalars an eligible dark matter candidate, in addition to triggering leptogenesis at the scale of the heavy neutrino masses. Taking two representative values of this mass scale, we identify the allowed regions of the parameter space of the model, which are consistent with all dark matter constraints. At the same time, the running of quartic couplings in the scalar potential to high scales is studied, thus subjecting the regions consistent with dark matter constraints to further requirements of vacuum stability, perturbativity and unitarity. It is found that part of the parameter space is consistent with all of these requirements all the way up to the Planck scale, and also yields the correct signal strength in the diphoton channel for the scalar observed at the Large Hadron Collider.
\end{abstract}

\section{Introduction}

The discovery of 'a Higgs-like boson' [1,2], apparently completes the Standard electroweak model (SM). However, it also compels us to mull over the necessity of physics beyond the SM. To mention one motivation for this, a SM Higgs of mass around $125 \mathrm{GeV}$, can break the absolute stability of vacuum at $10^{8-9} \mathrm{GeV}$, if the top quark mass $\left(M_{t}\right)$ and the strong coupling constant $\left(\alpha_{s}\right)$ are on the upper sides of their respective uncertainty bands. A recent next-to-next-to-leading order (NNLO) study [3] reveals that absolute stability up to the Planck scale requires

$$
M_{h}[\mathrm{GeV}]>129.4+1.4\left(\frac{\mathrm{M}_{\mathrm{t}}[\mathrm{GeV}]-173.1}{0.7}\right)-0.5\left(\frac{\alpha_{\mathrm{S}}\left(\mathrm{M}_{\mathrm{Z}}\right)-0.1184}{0.0007}\right) \pm 1.0_{\mathrm{th}}
$$

which, with all theoretical and experimental errors in $M_{t}$ and $\alpha_{s}$, implies that the absolute vacuum stability of SM is excluded at 95\% CL for $M_{h}<126 \mathrm{GeV}$. To compensate the negative contribution

\footnotetext{
${ }^{1}$ nabarunc@hri.res.in

${ }^{2}$ tpdkg@iacs.res.in

${ }^{3}$ biswarup@hri.res.in

4 tpis@iacs.res.in
} 
from the fermion loops (mainly the top quark loop), which rapidly drives the quartic self-coupling of Higgs to below zero, we need extra scalar loops that can ameliorate the problem. This provides one of the important motivations to consider a theory with new scalars which can make the vacuum stable up to high scales while having consistent results at low scales.

At the same time it is worthwhile to remember two rather pressing issues which prompt one to look beyond the Standard Model (BSM). These are the non-zero mass and mixing of neutrinos and the likely existence of Weakly Interacting Massive Particle(WIMP), contributing to Dark Matter (DM). While various Solar, atmospheric, reactor and accelerator-based experiments provide strong evidence for non-zero masses of neutrinos and mixing among their different flavors, several astrophysical and cosmological observations suggest the existence of some exotic particles that constitute the DM content of the universe. Given all this, it is an attractive idea to look for a theory that can simultaneously address all of the aforesaid problems in one framework. Here we consider one such scenario.

We investigate a model, first proposed in reference [4], that extends the SM with an extra Higgs doublet and three right handed neutrinos with a $Z_{2}$ symmetry, under which all SM particles are even while this additional scalar doublet and the right-handed neutrinos are odd. This symmetry prevents the additional doublet from having a vacuum expectation value(vev) thus vetoing the tree-level neutrino mass generation. Moreover, there exists a stable scalar particle in the form of the lightest neutral mass eigenstate of the additional doublet, which yields an eligible DM candidate. The extra doublet is essentially an Inert Doublet. Although a lot of study has already taken place on minimal Inert Doublet models [5-23], the extra appeal of this model lies in the radiative generation of neutrino mass. Though various aspects of this scenario have already been investigated [24-35], the present study includes the following points which have not been emphasized before.

- We investigate the vacuum stability of this model at various scales, and identify the regions of its parameter space, which keeps the model valid all the way unto the Planck scale. The contribution of additional scalar fields as well as the right-handed neutrinos to the renormalisation group (RG) equations [given in Appendix A] has been taken into account here. Using these modified RG equations we evaluate the scalar quartic couplings at different scales. During the evolution of the quartic couplings we demand not only vacuum stability but also perturbativity of the couplings as well as unitarity of the $2 \rightarrow 2$ scattering matrix at each scale.

- The heavy right-handed neutrinos introduce a new mass scale $(M)$ to the theory and the neutrino Yukawa couplings contribute to the RG evolution only at this mass scale. This brings out greater implications on the parameter space that distinguishes this model from minimal Inert doublet models. In this paper, we will show the salient features of the model that emerges from the above fact.

- This model also contributes to the study of leptogenesis due to this heavy right-handed fermions. The values of the right-handed neutrino mass scale used in the high-scale analysis are taken to be commensurate with leptogenesis constraints. Thus the part of the model space consistent unto the Planck scale is also supportive of leptogenesis.

- We also examine the candidature of the lightest $Z_{2}$-odd particle as DM candidate, and identify the allowed values of the couplings yield the right relic abundance. We ensure that the DM candidate is consistent with the recent result of direct detection experiments. We identify a substantial region of the parameter space, which simultaneously satisfy the vacuum stability, 
perturbativity and unitarity requirements and accommodate the DM candidate with the correct relic density.

- Finally, we examine the $125 \mathrm{GeV}$ scalar and make sure that the signal strengths in the observed channels (such as diphotons) are consistent with data from the Large Hadron Collider (LHC).

We organize the paper as follows. In Section 2, we briefly describe the model and its various features. In Section 3, we explain all the theoretical constraints and collider constraints that we use in the RG running of different quartic couplings. Next, in Section 4, we discuss the DM aspects of this model. After explaining our analysis strategy in Section 5, we present our results related to high-scale validity in Section 6. Finally in Section 7, we summarise our results.

\section{The Radiative Neutrino Mass Model with an Inert Doublet}

In addition to the SM fields, the radiative neutrino mass model with an inert doublet [4], contains a Higgs doublet $\left(\Phi_{2}\right)$ and three right handed $(\mathrm{RH})$ neutrinos $\left(N_{i}\right)$ with an unbroken $Z_{2}$ symmetry, under which the doublet and the right handed neutrinos are odd while all other SM particles are even. Being odd under the symmetry, $\Phi_{2}$ does not acquire any vacuum expectation value (vev) and has no tree-level couplings to fermions.

The relevant Yukawa and mass terms are

$$
-\mathcal{L}_{Y}=y_{i j} \bar{N}_{i} \tilde{\Phi}_{2}^{\dagger} \ell_{j}+h . c+\frac{M_{i}}{2}\left(\bar{N}_{i}^{c} N_{i}+h . c\right),(i, j=1-3)
$$

Here $\ell_{i}$ are the left-handed lepton doublets and $M_{i}$ are the Majorana mass term for the heavy righthanded neutrinos $N_{i}$

The scalar potential is

$$
\begin{aligned}
V & =\lambda_{1}\left(\Phi_{1}^{\dagger} \Phi_{1}\right)^{2}+\lambda_{2}\left(\Phi_{2}^{\dagger} \Phi_{2}\right)^{2}+\lambda_{3}\left(\Phi_{1}^{\dagger} \Phi_{1}\right)\left(\Phi_{2}^{\dagger} \Phi_{2}\right)+\lambda_{4}\left(\Phi_{2}^{\dagger} \Phi_{1}\right)\left(\Phi_{1}^{\dagger} \Phi_{2}\right) \\
& +\left[\frac{\lambda_{5}}{2}\left(\Phi_{1}^{\dagger} \Phi_{2}\right)^{2}+\text { h.c. }\right]+m_{\Phi_{1}}^{2} \Phi_{1}^{\dagger} \Phi_{1}+m_{\Phi_{2}}^{2} \Phi_{2}^{\dagger} \Phi_{2}
\end{aligned}
$$

where all parameters are real, and $\Phi_{1}$ is the SM Higgs doublet.

The two scalar doublets can be written as

$$
\Phi_{1}=\left(\begin{array}{c}
G^{+} \\
\frac{1}{\sqrt{2}}(v+h+i G)
\end{array}\right) \text { and, } \Phi_{2}=\left(\begin{array}{c}
H^{+} \\
\frac{1}{\sqrt{2}}(H+i A)
\end{array}\right)
$$

where, $v=246 \mathrm{GeV}$, is the electroweak vacuum expectation value (vev). One thus has five physical states $\left(h, H, A, H^{ \pm}\right)$and three Goldstone bosons $\left(G, G^{ \pm}\right)$. While $h$ corresponds to the physical SMlike Higgs field, the inert doublet components are one CP-even neutral scalar $(H)$, one CP-odd neutral $\operatorname{scalar}(A)$ and a pair of charged scalars $\left(H^{ \pm}\right)$. The physical masses are given by

$$
\begin{aligned}
M_{H^{ \pm}}^{2} & =m_{\Phi_{2}}^{2}+\frac{1}{2} \lambda_{3} v^{2} \\
M_{H}^{2} & =m_{\Phi_{2}}^{2}+\lambda_{L} v^{2}
\end{aligned}
$$




$$
M_{A}^{2}=m_{\Phi_{2}}^{2}+\lambda_{A} v^{2}
$$

where $\lambda_{L / A}=\frac{1}{2}\left(\lambda_{3}+\lambda_{4} \pm \lambda_{5}\right)$. The value of $\lambda_{1}$ is determined using $M_{h}=125 \mathrm{GeV}$.

Majorana masses for the light neutrinos are generated radiatively through one-loop exchange of the $Z_{2}$-odd neutral scalars. The general expression for the loop-induced contributions to the light neutrino mass matrix $[4]$ is

$$
\mathcal{M}_{i j}^{\nu}=\sum_{k=1}^{3} \frac{y_{i k} y_{j k} M_{k}}{16 \pi^{2}}\left[\frac{M_{H}^{2}}{M_{H}^{2}-M_{k}^{2}} \ln \frac{M_{H}^{2}}{M_{k}^{2}}-\frac{M_{A}^{2}}{M_{A}^{2}-M_{k}^{2}} \ln \frac{M_{A}^{2}}{M_{k}^{2}}\right]
$$

Thus, the neutrino masses and mixing are determined by the inert scalar masses and the right-handed neutrino masses $M_{i}$. These masses represent the scale of lepton number violation and hence that of leptogenesis $[36,37]$ in this model. Some studies have already been done in this context $[11,30,32,33$, 38]. Our choice of the right-handed neutrino (Majorana) mass scales made our study automatically compatible with leptogenesis. To satisfy the necessary constraints in the low DM mass region where $M_{D M}<M_{W}$, one must take the lightest of the Majorana masses to be $M \geq 110 \mathrm{TeV}$, where as in the high DM mass region $\left(M_{D M}>500 \mathrm{GeV}\right)$ the bound is only $1 \mathrm{TeV}$ [11]. Hence, to be consistent in both cases, we use two values of $\mathrm{M}$, (a) $M=110 \mathrm{TeV}$ and (b) $M=10^{9} \mathrm{TeV}$. While, in one hand, choice of (a) is motivated by the idea of having the lowest possible leptogenesis scale, we choose to work with (b) which have interesting consequences on the RG runnning. We will show in later sections, how the mass scale (b) of $M$ affects the stability of the vacuum mainly in the high DM mass region and eventually explain the physical reasons behind it.

Along with the above restrictions we also demand $M_{\nu} \sim \mathcal{O}(0.1 \mathrm{eV})$ to be consistent with neutrino oscillation data for some fixed $M$ and other exotic scalar masses. However, for simplicity, we consider only one diagonal Yukawa coupling $\left(y_{\nu}\right)$ and do not look into the hierarchical details of the Yukawa matrix. At this point, it is to be noted that a more rigorous study with the intricate flavor structure of the neutrino Yukawa matrix can highlight the region of the model space that fits the observed pattern of neutrino mixing [35]. However, we would like to emphasize that the broad conclusions on the high-scale validity of this scenario vis-a-vis the DM constraints remain unchanged. Finally, we should mention that the lighter state between $\mathrm{H}$ and $\mathrm{A}$ is the DM candidate. We present our illustrative results for cases where $\mathrm{H}$ plays this role.

\section{Constraints from perturbativity, unitarity, vacuum stability and collider data}

In this section, we briefly describe the constraints that are imposed on the model parameters and how exactly they shape the results so obtained.

\subsection{Vacuum stability}

The scalar potential is considered bounded from below, if it does not turn negative for large field values along all possible field directions. In this model, stability of the electroweak vacuum is ensured up to some specified energy scale if the following conditions are satisfied for all scales $Q$ up to that scale:

$$
\text { vsc1 : } \quad \lambda_{1}(Q)>0
$$




$$
\begin{array}{lll}
\operatorname{vsc} 2 & : & \lambda_{2}(Q)>0 \\
\operatorname{vsc} 3: & \lambda_{3}(Q)+\sqrt{\lambda_{1}(Q) \lambda_{2}(Q)}>0 \\
\operatorname{vsc} 4: & \lambda_{3}(Q)+\lambda_{4}(Q)-\left|\lambda_{5}(Q)\right|+\sqrt{\lambda_{1}(Q) \lambda_{2}(Q)}>0
\end{array}
$$

Such conditions have been elaborately discussed in literature [39-42]. One should make a note that these conditions ensure absolute stability of the electroweak vacuum. For metastability, the conditions are somewhat less stringent.

\subsection{Perturbativity}

For the scalar quartic coupling $\lambda_{i}(i=1-5)$, the criterion for perturbativity is,

$$
\lambda_{i}(Q)<4 \pi
$$

The corresponding constraints for the Yukawa and gauge interactions are,

$$
y_{i}(Q), g_{i}(Q)<\sqrt{4 \pi}
$$

where, Q represents the energy scale at which they are being computed. We demand that the criteria in Eq. 8 be obeyed at all energy scales up to the cut-off of this model.

\subsection{Unitarity}

A further set of conditions come on demanding unitarity of the scattering matrix comprising all $2 \rightarrow 2$ channels involving, by the optical theorem [43-46]. In our context, this translates into the condition that each distinct eigenvalue of the aforementioned amplitude matrix be bounded above at $8 \pi$ (after factoring out $\frac{1}{16 \pi}$ from the matrix). These eigenvalues are:

$$
\begin{aligned}
a_{ \pm} & =\frac{3}{2}\left(\lambda_{1}+\lambda_{2}\right) \pm \sqrt{\frac{9}{2}\left(\lambda_{1}-\lambda_{2}\right)^{2}+\left(2 \lambda_{3}+\lambda_{4}\right)^{2}} \\
b_{ \pm} & =\frac{1}{2}\left(\lambda_{1}+\lambda_{2}\right) \pm \sqrt{\frac{1}{4}\left(\lambda_{1}-\lambda_{2}\right)^{2}+\lambda_{5}^{2}} \\
c_{ \pm} & =d_{ \pm}=\frac{1}{2}\left(\lambda_{1}+\lambda_{2}\right) \pm \sqrt{\frac{1}{4}\left(\lambda_{1}-\lambda_{2}\right)^{2}+\lambda_{5}^{2}} \\
e_{1} & =\left(\lambda_{3}+2 \lambda_{4}-3 \lambda_{5}\right), \\
e_{2} & =\left(\lambda_{3}-\lambda_{5}\right), \\
f_{1} & =f_{2}=\left(\lambda_{3}+\lambda_{4}\right), \\
f_{+} & =\left(\lambda_{3}+2 \lambda_{4}+3 \lambda_{5}\right), \\
f_{-} & =\left(\lambda_{3}+\lambda_{5}\right) .
\end{aligned}
$$

\subsection{Collider data}

In addition to the theoretical constraints discussed above, important bounds on scalar mass parameters come from collider data. 
- In order to identify $h$ with the SM-like Higgs as observed by the ATLAS and CMS collaborations, one requires $M_{h} \simeq 125 \mathrm{GeV}$.

- To be consistent with the LEP bounds, one must have

$$
\begin{aligned}
M_{H}+M_{A} & >M_{Z}, \\
M_{H^{ \pm}}+M_{A / H} & >M_{W} .
\end{aligned}
$$

Moreover, for neutralino as in the supersymmetric context, LEP-II searches limit pseudo-scalar mass $\left(M_{A}\right)$ to $100 \mathrm{GeV}$ when $M_{H}<M_{A}[8,47]$. Similarly, chargino search data, properly extrapolated, imply $M_{H^{ \pm}}>70 \mathrm{GeV}$ [48].

- Though all the tree-level couplings of $h$ are identical to those of the SM Higgs, the charged scalar $H^{ \pm}$potentially modifies the loop induced couplings $h \gamma \gamma$ and $h Z \gamma$ via loop contributions [49-53]. We theoretically compute the signal strength $\mu_{\gamma \gamma}$ for $h$ decaying to the diphoton channel as the ratio of the decay width in the 'model' to that in the SM. Its experimental value reported by the ATLAS and CMS collaborations stand at $1.17 \pm 0.27$ and $1.13 \pm 0.24$ respectively [54,55]. Demanding the signal strength to be within $2 \sigma$ limits of the experimentally quoted values puts further constraints on the model. We use the limit on $\mu_{\gamma \gamma}$ weighted as below:

$$
\begin{gathered}
\frac{1}{\sigma^{2}}=\left(\frac{1}{\sigma^{2}}\right)_{A T L A S}+\left(\frac{1}{\sigma^{2}}\right)_{C M S} \\
\frac{\mu_{\gamma \gamma}}{\sigma^{2}}=\left(\frac{\mu_{\gamma \gamma}}{\sigma^{2}}\right)_{A T L A S}+\left(\frac{\mu_{\gamma \gamma}}{\sigma^{2}}\right)_{C M S}
\end{gathered}
$$

where, the numerators on the right hand side denotes the central values of the respective experimental results and $\sigma$ are the corresponding uncertainties.

\section{Dark Matter Issues}

As stated earlier, we identify $H$ as the DM candidate. For complimentary choice, namely, $A$ with the same mass as the DM candidate, we have checked that the contribution to the relic density is of similar magnitude. The relevant DM constraints to be considered are as follows:

- According to recent PLANCK experiment [56] the observed cold DM relic density is

$$
\Omega_{\mathrm{DM}} h^{2}=0.1199 \pm 0.0027
$$

We restrict our result up to $3 \sigma$ deviation from the central value.

- Strong constraints come from direct DM searches. Recently, XENON100 [57] and LUX [58] experiments have put upper-bound on the DM-nucleon scattering cross-section for a wide range of the DM mass. In our case, the direct detection cross-section strategy is based on t-channel Higgs mediation. We choose to work in the region of the parameter space allowed by the LUX limit.

- For $M_{H} \leq M_{h} / 2$, the decay mode of Higgs to two DM particle $(h \rightarrow H H)$ will presumably contribute to the invisible decay width of the Higgs boson. We take into account the current constraint on the Higgs invisible branching ratio $(<20 \%)$ from model independent Higgs precision analysis $[59,60]$. 
- Our parameter scan has included situations where the $Z_{2}$-odd scalar and the pseudo-scalar are close by in mass and with co-annihilation yield correct relic abundance within the $\pm 3 \sigma$ of the observed values when the DM mass is high $(>500 \mathrm{GeV})$. For DM mass in between $100 \mathrm{GeV}$ and $500 \mathrm{GeV}$, there is prima facie the possibility of co-annihilation between the spinless DM candidate and a right-handed neutrino which can give rise to correct relic abundance, as discussed in Ref. [61]. In our case, however, such a possibility is ruled out by the additional requirement of leptogenesis, which in turn puts a lower bound on the mass range for the right-handed neutrino(s).

Hence, only the following two cases survive all the above constraints.

\subsection{Case-A: Low mass DM $\left(50 \mathrm{GeV}<\mathrm{M}_{\mathrm{H}}<90 \mathrm{GeV}\right)$}

In this mass region, the dominant annihilation channel for $\mathrm{H}$ goes to the DM self-annihilation processes through $h$ mediation. This keeps the relic density at the right level. For both positive and negative values of DM-DM-Higgs couplings, the relic density remains within the allowed range as long as the $M_{H}<90 \mathrm{GeV}$. The sub-dominant contribution to the relic density comes from the t-channel processes to vector boson final states mediated by $A$ and $H^{ \pm}$. A detailed discussion in this regard on a similar model can be found, for example in $[11,13,22]$. However, the coupling $\lambda_{2}$ has no effect in the relic density calculation. In the next section, we will discuss this results elaborately. One more notable point is that, annihilation processes that mediated by the heavy right-handed neutrinos give negligible contribution (less than 1\%) to the relic density calculation. These processes are suppressed by the heavy mediator mass.

\subsection{Case-B: High mass DM $\left(M_{H}>500 \mathrm{GeV}\right)$}

The interesting feature of this region is that, the correct relic abundance can be achieved if and only if $H, A$ and $H^{ \pm}$are almost degenerate, at most have a mass difference of the order of $10 \mathrm{GeV}$. This is mainly because at this high mass, the annihilation channels with vector boson final states can have direct quartic couplings $\left(H H V V, V=W^{ \pm}, Z\right)$ or can be mediated by any of the three scalars through $t / u$ channels. These diagrams yield too large an annihilation cross-section to match with the proper relic density. However, cancellation between direct quartic coupling diagrams and $t / u$ channels diagram occurs for mass-degenerate $H, A$ and $H^{ \pm}$, which in turn brings down the annihilation cross-section to the desired range.

\section{$5 \quad$ Analysis strategy}

The aim of this study is to probe the parameter space of an inert doublet model (IDM) augmented with heavy RH neutrinos compatible with various theoretical and experimental constraints elaborated in the previous sections. We carry out our investigation in the two separate mass regions. In each region, we scan over the relevant parameters, namely, the masses $M_{H}, M_{A}$ and $M_{H}^{ \pm}$, and the coupling $\lambda_{L}$. With $M_{h}$ fixed at $125 \mathrm{GeV}, \lambda_{2}, \lambda_{L}, M_{H}, M_{A}$ and $M_{H}^{ \pm}$fix all the remaining quartic interactions. At this point it is to be mentioned that the parameter $\lambda_{2}$ can not be constrained by any physical observable at least at tree level. However, $\lambda_{2}$ is bounded by the stability and perturbativity condition 
and we have explicitly checked that $0<\lambda_{2}<0.36$ to satisfy the theoretical constraints. Rather than scanning over $\lambda_{2}$, we have demonstrated our results with two benchmark values (0.1 and 0.001) for it, both of which are well within the above limit.

These quartic couplings are then used as the electroweak boundary conditions at $Q=M_{t}$ and their RG evolution to high scales is studied. Here $M_{t}$ denotes the top quark pole mass. The reader is reminded that the effect of the $\mathrm{RH}$ neutrinos is turned on at a scale $Q=M$. Thus, for $M_{t} \leq Q \leq M$, we do not include the RH neutrino contributions to the one-loop RG equations. We include such conditions for $Q \geq M$ and use the RG equations listed in the appendix. The scale up to which the scenario remains consistent is denoted by $\Lambda_{U V}$. For a generic parameter point $\lambda_{i}\left(Q=M_{t}\right)$, we check the aforementioned theoretical constraints at all intermediate scales up to $\Lambda_{U V}$. If the constraints are all satisfied, we tag $\lambda_{i}\left(Q=M_{t}\right)$ as an allowed point. This marks out an allowed region in the parameter space defined at the electroweak scale. Moreover, the effects of constraints stemming from the DM observables and collider searches are examined independently in this region. The finally allowed parameter regions are thus identified and presented for benchmark values of $\lambda_{2}$ and $M$. We use the publicly available package micrOMEGAs-v3.6.9.2 [62] for DM analysis. In all the cases, we also make sure that our allowed parameter space does not violate the oblique T-parameter constraints [63, 64].

Amongst the SM fermions, only the top quark plays the dominant role in the evolution of the couplings. The boundary condition for its Yukawa interaction at the electroweak scale is fixed by $y_{t}\left(M_{t}\right)=$ $\frac{\sqrt{2} M_{t}}{v}\left(1-\frac{4}{3 \pi} \alpha_{s}\left(M_{t}\right)\right)$. We have used $M_{t}=173.39 \mathrm{GeV}$ throughout our analysis.

\section{$6 \quad$ Numerical results}

\subsection{Low mass DM region}

We perform a detailed parameter space scan where $M_{H}<100 \mathrm{GeV}$. In this scan, we impose the LEP bounds as discussed in Sec 3.4.

$$
\begin{array}{r}
\lambda_{L}:[-1.0,1.0] \\
M_{H}:[50.0 \mathrm{GeV}, 90 . \mathrm{GeV}] \\
M_{A}:[100 . \mathrm{GeV}, 500 . \mathrm{GeV}] \\
M_{H^{+}}:[100 . \mathrm{GeV}, 500 . \mathrm{GeV}]
\end{array}
$$

We solve the RG equations for two values of $M, 110 \mathrm{TeV}$ and $10^{9} \mathrm{TeV}$ respectively. We then show the allowed parameter space in the $\lambda_{L}-M_{H}$ plane for different choices of $\Lambda_{U V}$ in Fig 1 and 2. The regions denoted by A (red), B (cyan) and C (green) denote $\Lambda_{U V}=10^{6}, 10^{16}$ and $10^{19} \mathrm{GeV}$ respectively. We overlay the region allowed by the Higgs to diphoton signal strength within $2 \sigma$ limits of the current data as grey region named D. As mentioned earlier, the full analysis is done for two values of $\lambda_{2}$ at the electroweak scale (0.1 and 0.001).

Let us briefly summarize the features that emerges from the figures and their possible explanations.

- From Fig.1, it can be seen that an Inert doublet model with right handed neutrinos posses stable vacuum even up to the Planck scale, but the higher the cut-off scale $\Lambda_{U V}$, the less amount of 


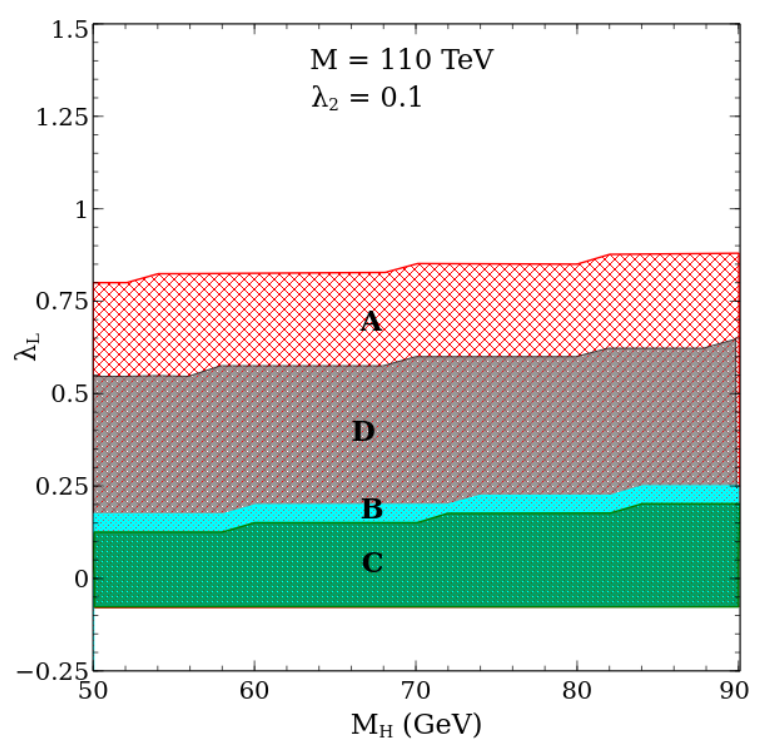

(a)

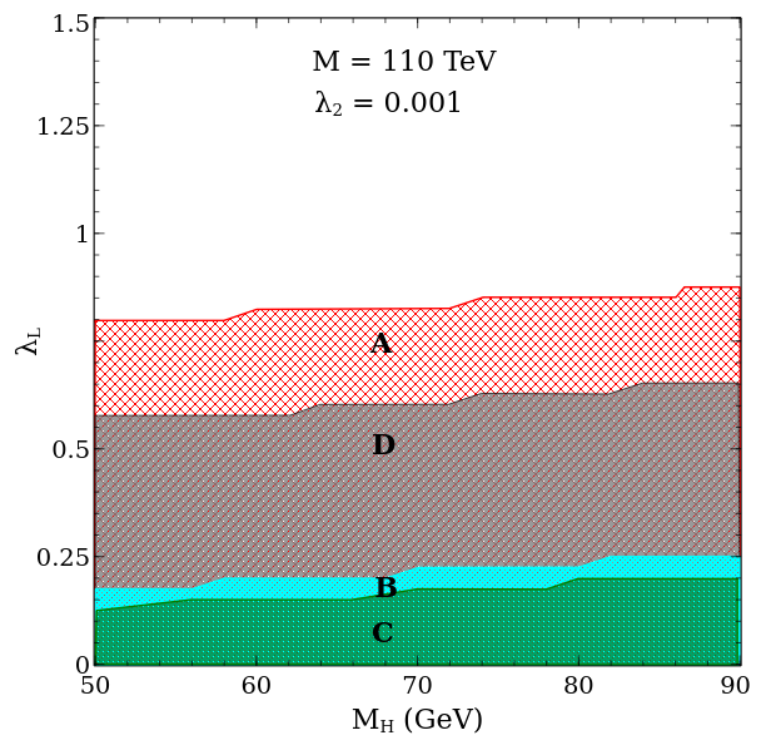

(c)

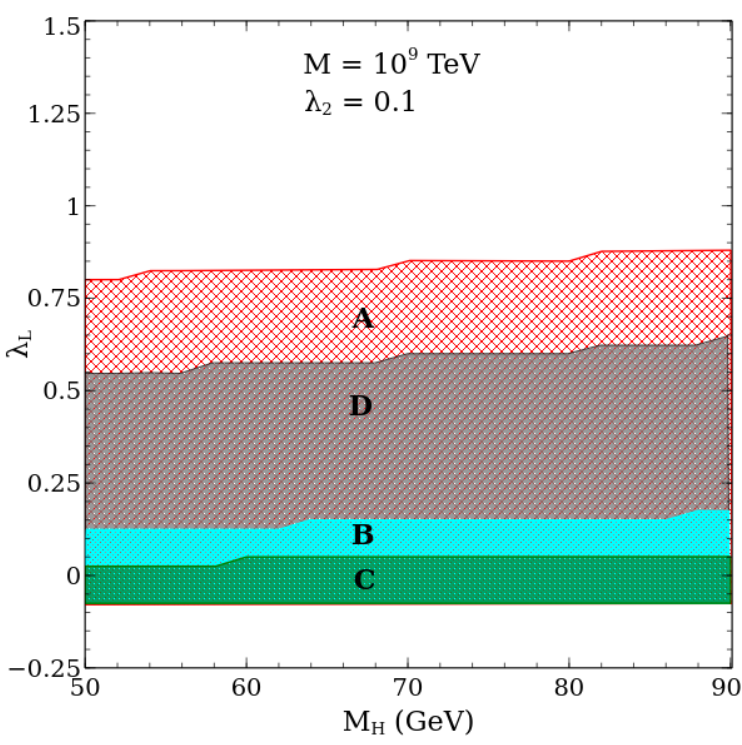

(b)

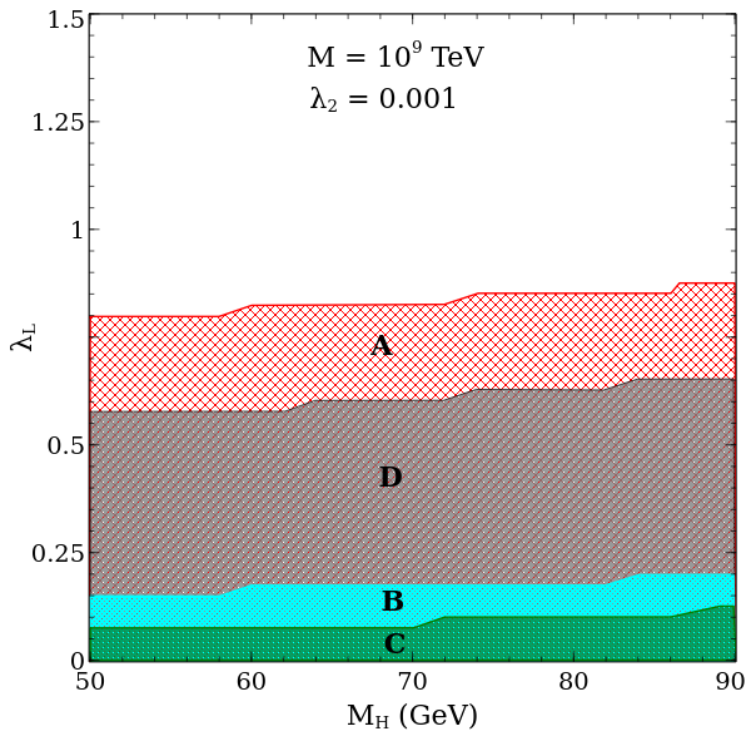

(d)

Figure 1: Regions compatible with the theoretical constraints in the $M_{H^{-}} \lambda_{L}$ plane for $M=110 \mathrm{TeV}$ (left panel) and $10^{9} \mathrm{TeV}$ (right panel) with three different choices of $\Lambda_{U V}$ and two values of $\lambda_{2}$ (upper and lower panel). The regions denoted by A (red), B (cyan) and C (green) obey these constraints up to $\Lambda_{U V}=10^{6}, 10^{16}$ and $10^{19} \mathrm{GeV}$, respectively. The grey region denoted by D keeps the Higgs to diphoton signal strength within $2 \sigma$ limits of the current data.

parameter space becomes allowed. To understand this, one can recollect that the top quark Yukawa coupling in the SM is responsible for the downward evolution of the scalar self-coupling, which poses a threat to the vacuum stability. The presence of the additional scalar quartic 
couplings $\left(\lambda^{\prime} s\right)$ in a model like this offsets such an effect; however, the boost thus provided to these couplings tend to violate the perturbativity and unitarity condition. This necessitates a tightrope walking, and the scale up to which it is possible is $\Lambda_{U V}$. Hence, it is natural that for higher $\Lambda_{U V}$, fewer combinations of parameters will achieve this fine balance, and consequently the allowed region shrinks.

- Fig.1 also shows that $\lambda_{L}$ is bounded on both sides and the limits stay almost same for different right handed neutrino mass scale $(M)$. This is of no surprise and can be easily understood. Since $\lambda_{3}=\frac{2}{v^{2}}\left(M_{H^{ \pm}}^{2}-M_{H}^{2}+\lambda_{L} v^{2}\right)$, the upper bound on $\lambda_{L}$ is imposed by the requirement of perturbative unitarity. This is because a higher positive value of $\lambda_{L}$ makes $\lambda_{3}$ large at the electroweak scale which violates perturbative unitarity in the course of its evolution under RG. On the other hand, a large negative value of $\lambda_{L}$ induces a large negative value to $\lambda_{3}$. As a consequence, the vacuum stability condition vsc4 of Eq.7d is violated even near the electroweak(EW) scale. This puts a lower limit on $\lambda_{L}$ independent of $M$ and $\Lambda_{U V}$, as evident from Fig.1. However, it must be noted that the lower limit of $\lambda_{L}$ is not independent of $\lambda_{2}$, which is another consequence of Eq.7d that requires the condition $\lambda_{3}+\lambda_{4}-\left|\lambda_{5}\right|>-\sqrt{\lambda_{1} \lambda_{2}}$ to be satisfied. With the decrease in the value of $\lambda_{2}$ the lower limit of the combination $\lambda_{3}+\lambda_{4}-\left|\lambda_{5}\right|$ enhances. This combination can be approximated to $\lambda_{L}$ in the perturbative regime and hence lower values of $\lambda_{2}$ increases the lower bound of $\lambda_{L}$, as can be seen from the upper and lower panel of Fig.1 that corresponds to $\lambda_{2}=0.1$ and $\lambda_{2}=0.001$ respectively.

- To check the compatibility of the DM constraints with the theoretical ones, we look for the region allowed by the $3 \sigma$ limits on $\Omega_{\mathrm{DM}} h^{2}$ from PLANCK data and 90\% CL limit on the spinindependent DM-nucleon scattering cross-section from LUX data. In Fig. 2, we show the parameter space allowed by the entire set of DM constraints. An inspection of Fig. 1 and Fig. 2 shows that almost the full parameter space allowed by the DM constraints lies in the region which is also favoured by the vacuum stability condition all the way up to the Planck scale. However, the region corresponding to $\lambda_{L} \leq-0.1$ (shaded region in Fig. 2) does not lead to the stable vacuum.

- It should be noted that in Fig. 2 we keep $M_{H}^{ \pm}$and $M_{A}$ fixed at $200 \mathrm{GeV}$. For higher values of $M_{H^{ \pm}}$and $M_{A}$, there is hardly any change in the annihilation cross-section. However, for values of $M_{H}^{ \pm}$and $M_{A}$ less than $200 \mathrm{GeV}$, the allowed region of Fig. 2 gets slightly modified. For example, for $M_{H} \simeq 70 \mathrm{GeV}$ and $M_{H^{ \pm}}=M_{A}=200 \mathrm{GeV}$, the DM-DM-Higgs coupling $\lambda_{L} \simeq 0.007$, but for $M_{H^{ \pm}}=M_{A}=100 \mathrm{GeV}$, one needs $\lambda_{L} \simeq 0.009$ to satisfy the relic density constraint. It should be noted however that both of the above points in the parameter space are within the stability region as shown in Fig. 1.

Therefore, it is not possible to constrain $M_{H}^{ \pm}$and $M_{A}$ using DM constraints alone, theoretical constraints however predict strong upper bounds on these masses, as is evident from Fig. 3 and Fig. 4.

- In Fig. 3 and Fig. 4, we show the theoretically valid regions in the $\lambda_{3}-M_{H}^{ \pm}$and $\lambda_{L}-M_{A}$ planes. As in previous cases, we exhibit the same for two different $M$ and $\lambda_{2}$ values. Also, in each case, we overlay the parameter spaces allowed by the $2 \sigma$ limit of Higgs to diphoton signal strength (region D). We observe that a tight upper bound of $160-170 \mathrm{GeV}$ is realised on the masses $M_{H}^{ \pm}$ and $M_{A}$ for the cut-off at the Planck scale and the couplings $\left(\lambda_{3}, \lambda_{L}\right)$ are also bounded. The upper bounds on the masses $M_{H}^{ \pm}$and $M_{A}$ are imposed by the requirement of perturbativity and unitarity up to the desired cut-off. With $M_{H}$ in the aforementioned range, large masses of the 


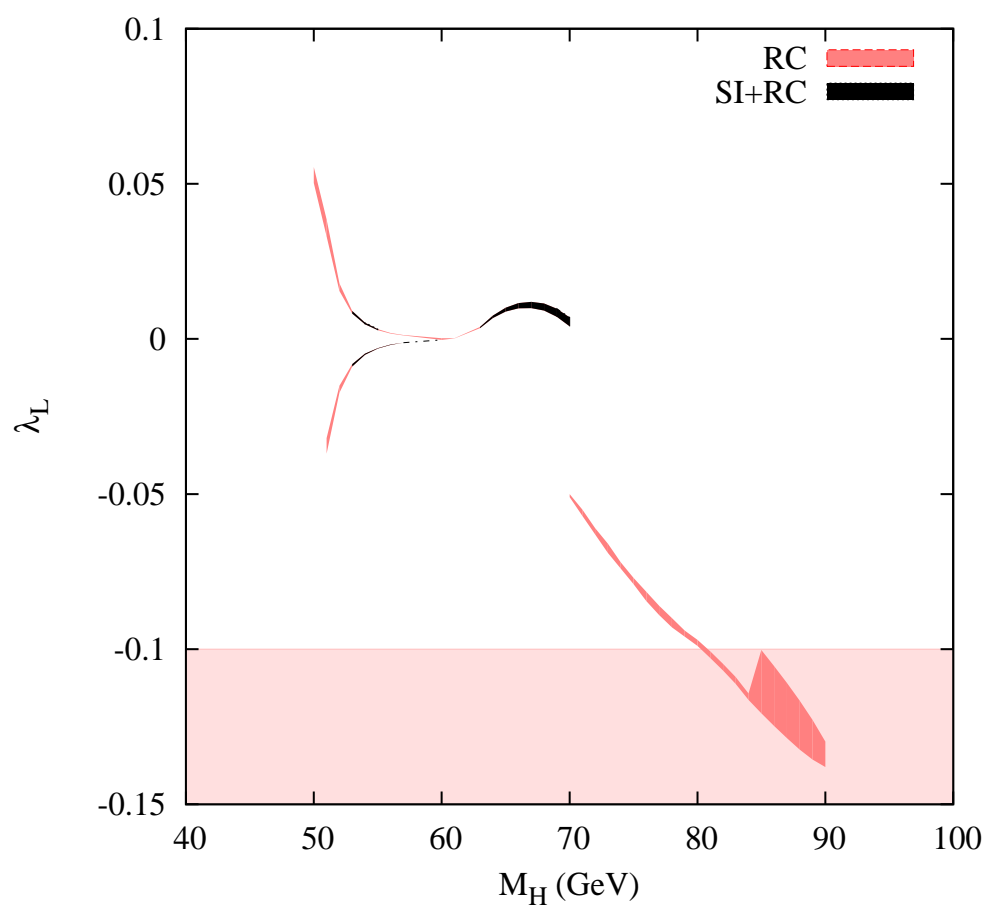

Figure 2: Region allowed by imposing the constraints on relic density $(\mathrm{RC})$ and spin-independent cross-section(SI) for DM-nucleon scattering. The red(gray) region is allowed only by the requirement of $\Omega_{\mathrm{DM}} h^{2}$ being in the correct range. The black region is allowed by both the $\Omega_{\mathrm{DM}} h^{2}$ and direct detection constraints. The shaded horizontal band below is disallowed by vacuum stability conditions. Here, $M_{H^{ \pm}}=M_{A}=200 \mathrm{GeV}$.

other $Z_{2}$-odd scalars imply high values of the quartic couplings at the electroweak scale which potentially violate perturbativity or unitarity at some high scale. On the other hand, the vacuum stability condition $\lambda_{3}+\sqrt{\lambda_{1} \lambda_{2}} \geq 0$ forbids large negative values of $\lambda_{3}$ and perturbativity puts the upper bound. The explanations of the limits of $\lambda_{L}$ have already been mentioned above.

- The new physics contribution to the Higgs to diphoton decay channel comes from the charged Higgs loop which is a function of $M_{H^{ \pm}}$and $\lambda_{3}$. The fact that $\lambda_{3}$ can not be large negative results in a fall in the signal strength for diphoton channel. However, the parameter space for $\Lambda_{U V}=10^{19} \mathrm{GeV}$ is still allowed by the current limits on $\mu_{\gamma \gamma}$ defined in Eq. 13.

\subsection{High mass DM region}

This section demonstrates the high scale validity of our scenario in the limit of a high DM mass. As discussed earlier, one needs to tune the mass splitting amongst $H, H^{ \pm}$and $A$ and the coupling $\lambda_{L}$ to an appropriate degree in order to achieve a relic density within the desired bounds. It is seen that the maximal allowed splitting $(\Delta M)$ amongst the masses of the $Z_{2}$ odd scalars is $10 \mathrm{GeV}$. As previously mentioned, for each chosen values of $\lambda_{2}$ (0.1 and 0.001), one is thus motivated to scan the high DM 


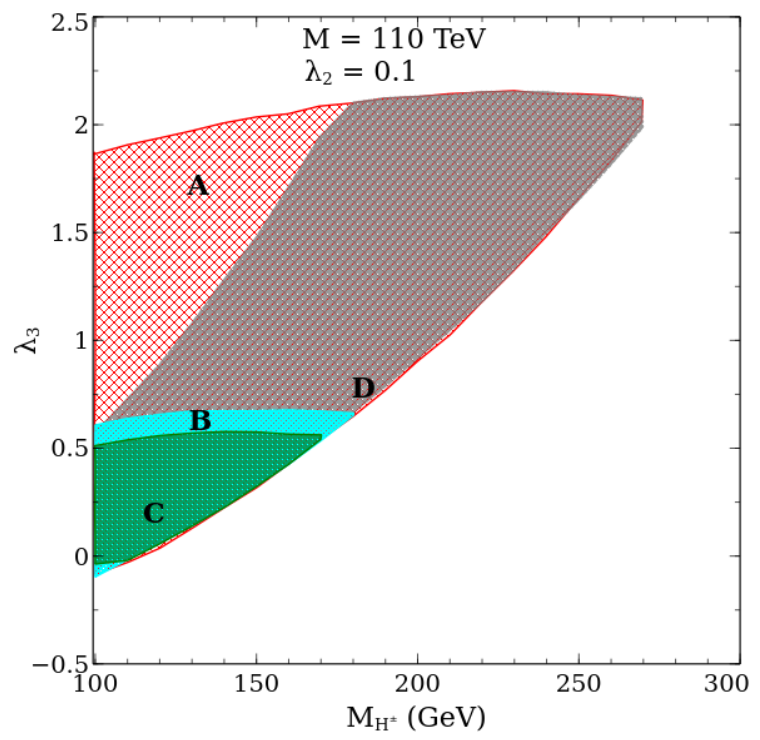

(a)

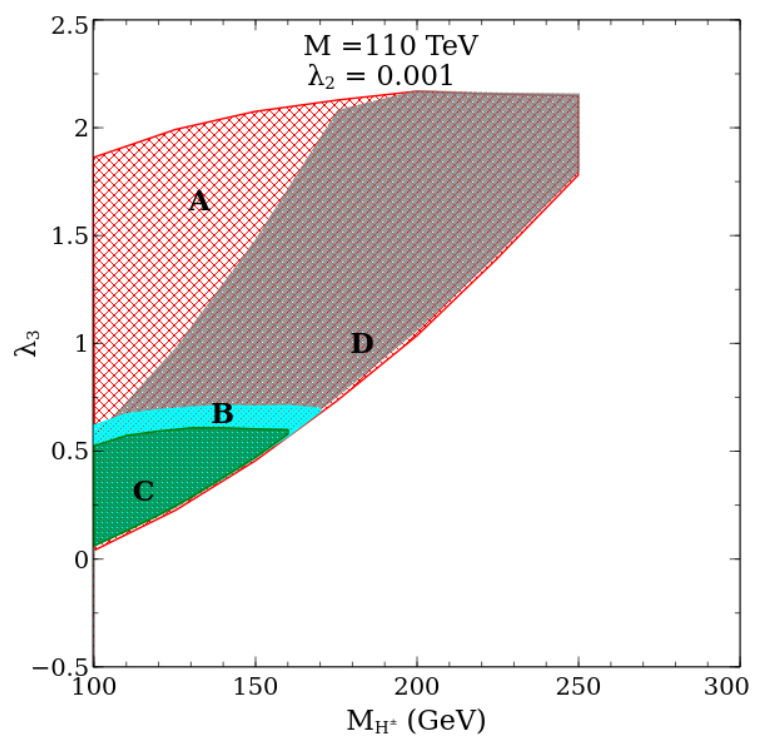

(c)

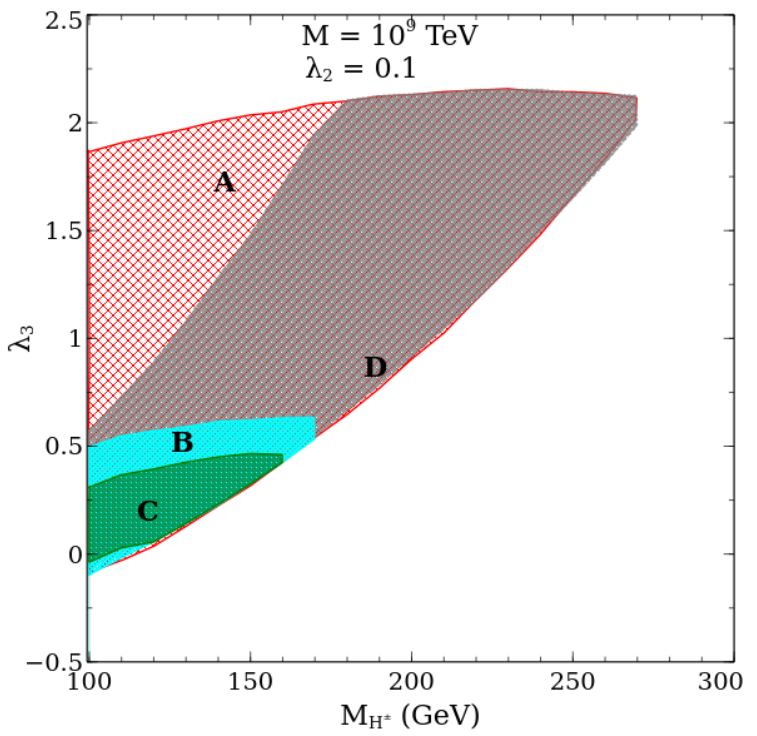

(b)

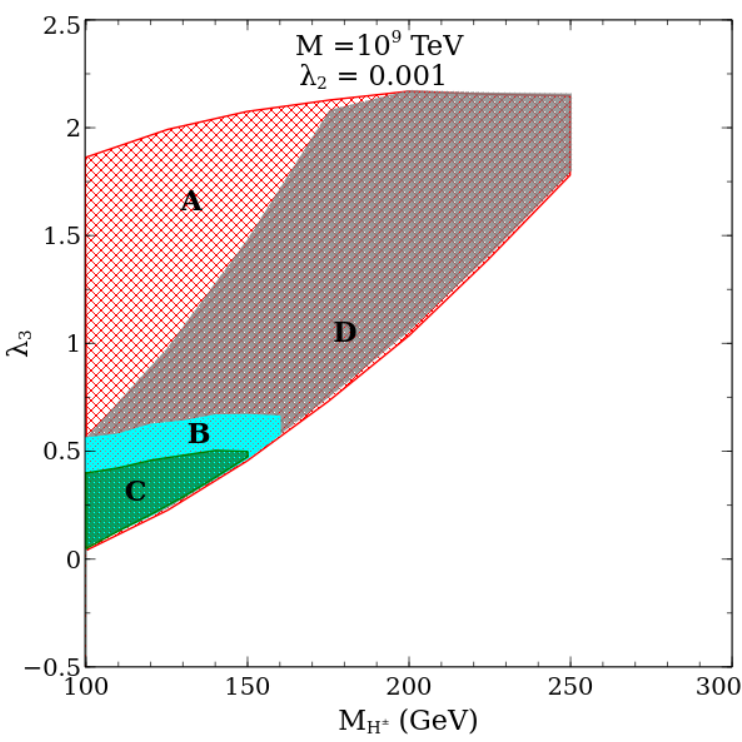

(d)

Figure 3: Regions allowed by the theoretical constraints projected in the $M_{H}^{ \pm}-\lambda_{3}$ plane for $M=110 \mathrm{TeV}$ (left panel) and $10^{9} \mathrm{TeV}$ (right panel) with three different choices of $\Lambda_{U V}$ and two values of $\lambda_{2}$ (upper and lower panel). The regions denoted by A (red), B (cyan) and C (green) obey those constraints up to $\Lambda_{U V}=10^{6}, 10^{16}$ and $10^{19} \mathrm{GeV}$, respectively. The grey region denoted by $\mathrm{D}$ shows the $2 \sigma$ allowed limit of the Higgs to diphoton signal strength. 


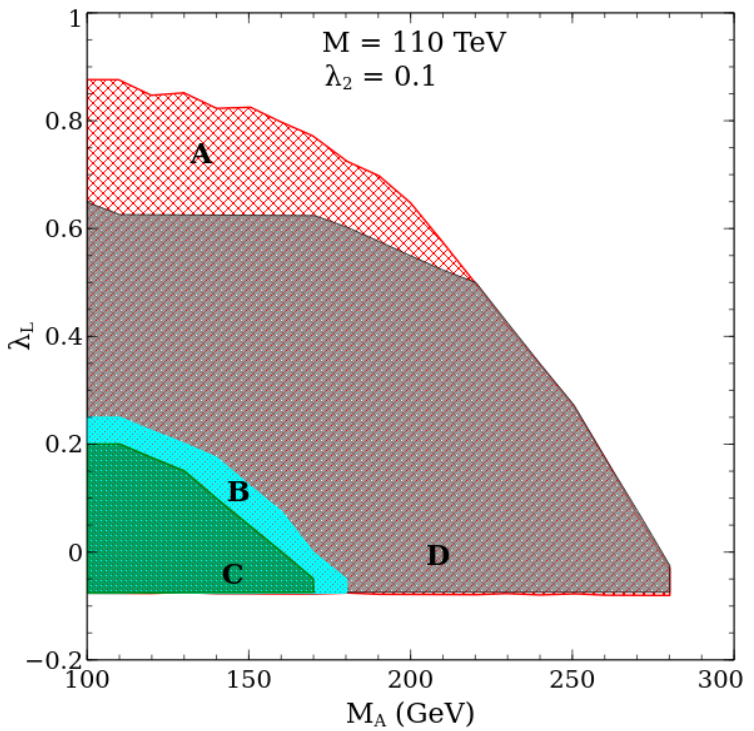

(a)

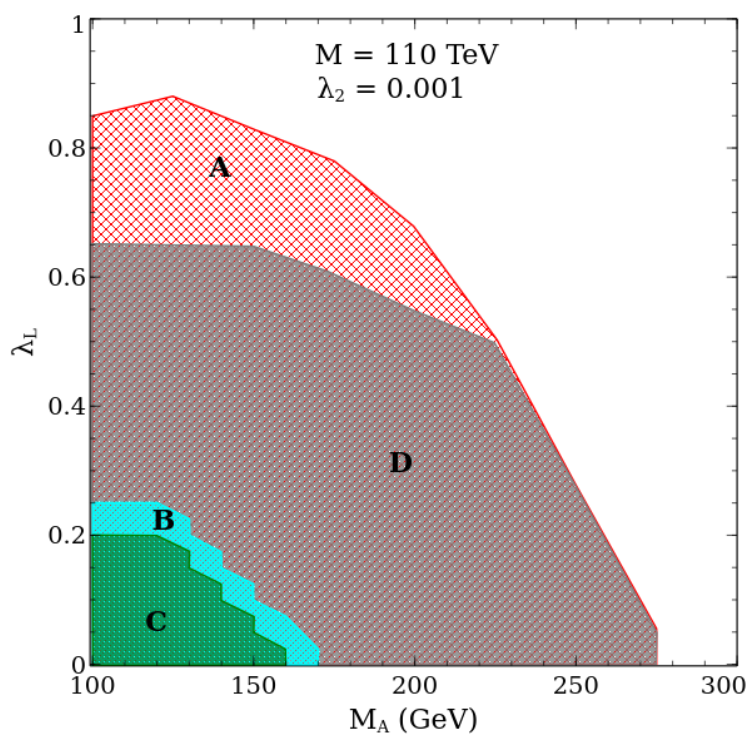

(c)

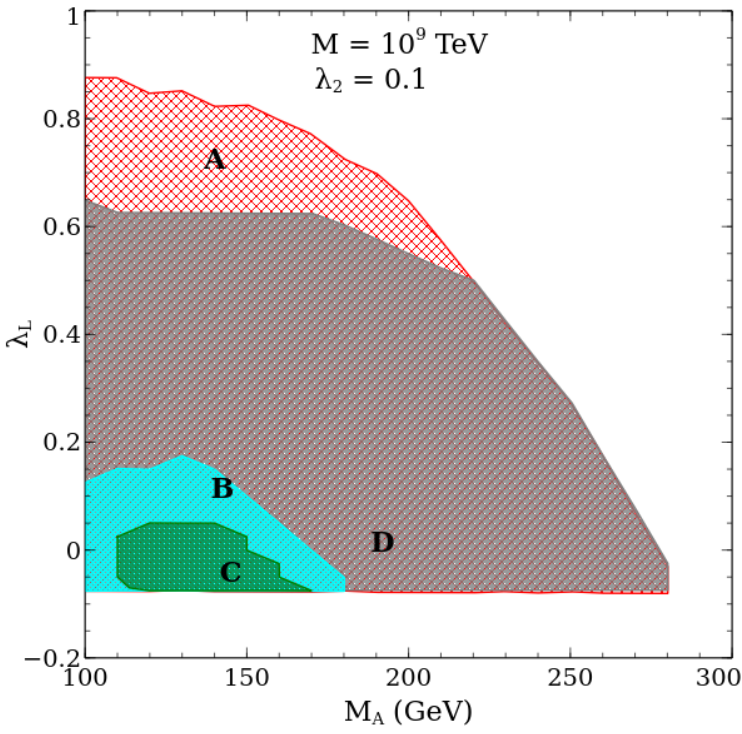

(b)

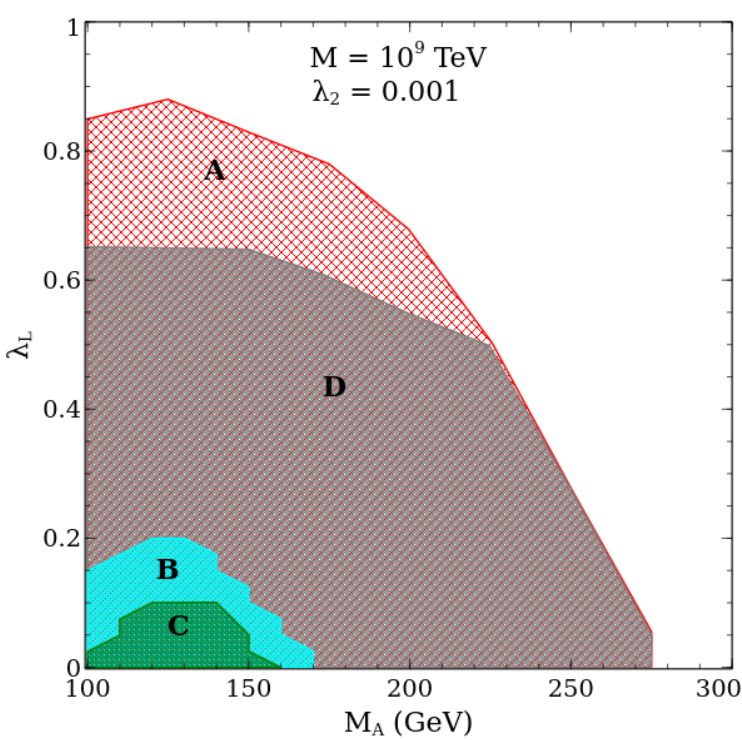

(d)

Figure 4: Regions allowed by the theoretical constraints projected in the $M_{A}-\lambda_{L}$ plane for $M=110 \mathrm{TeV}$ (left panel) and $10^{9} \mathrm{TeV}$ (right panel) with three different choices of $\Lambda_{U V}$ and two values of $\lambda_{2}$ (upper and lower panel). The regions denoted by A (red), B (cyan) and C (green) obey those constraints up to $\Lambda_{U V}=10^{6}, 10^{16}$ and $10^{19} \mathrm{GeV}$, respectively. The grey region denoted by $\mathrm{D}$ shows the $2 \sigma$ allowed limit of the Higgs to diphoton signal strength. 
mass region in the following ranges:

$$
\begin{array}{r}
\lambda_{L}:[-1.0,1.0] \\
M_{H}:[550.0 \mathrm{GeV}, 1000.0 \mathrm{GeV}] \\
M_{A}:\left[M_{H}, M_{H}+10.0 \mathrm{GeV}\right] \\
M_{H^{+}}:\left[M_{H}, M_{H}+10.0 \mathrm{GeV}\right]
\end{array}
$$

Unlike the previous case, while the theoretical constraints ruled out a large portion of the parameter space, the DM constraints put a less stringent bound on it in this high DM mass region. Therefore, in Fig. 5 and Fig. 6, we demonstrate the parameter region allowed only by the DM constraints and then overlay the part which are further allowed by theoretical constraints. The full (pink) region denoted by $\mathbf{R C}+$ SI shows the valid parameter space allowed by DM constraints. In accordance with previous notation, the regions denoted by A (red), B (cyan) and C (green) denote $\Lambda_{U V}=10^{6}, 10^{16}$ and $10^{19}$ $\mathrm{GeV}$ respectively.

Let us explain the various features of the model that emerges from the figures, in detail.

(1) The interplay of the theoretical and experimental constraints is studied in the form of correlation plots in the $M_{H}-\lambda_{L}$ (Fig. 5) as well as $M_{H^{ \pm}}-\lambda_{3}$ (Fig. 6) plane. As can be seen, a significant amount of parameter space is forbidden for the theory to be valid until the Planck scale.

(2) Similar to the previous section, the upper and lower bounds on $\lambda_{L}$ are placed from the requirements of perturbative unitarity and vacuum stability conditions respectively, as shown in Fig. 5. In this region too the lower limit of $\lambda_{L}$ has dependence on $\lambda_{2}$. The same happens for $\lambda_{3}$, as depicted in Fig. 6.

(3) It is to be noted that the theoretically allowed parameter space in this high DM mass region is fully consistent with the Higgs to diphoton LHC data. Since, a heavy $H^{ \pm}$which naturally arises in this region, does not cause any significant deviation in diphoton signal strength for the SM-like scalar. This occurs even with a large positive $\lambda_{3}$ (within the bounds shown in Fig. 6). In principle, a large negative $\lambda_{3}$ could modify $\mu_{\gamma \gamma}$ unacceptably. However, as Fig. 6 shows, such values are inconsistent with the aforementioned theoretical constraints.

(4) It is worth noting here that the parameter space valid until the Planck scale and corresponding to $M=110 \mathrm{TeV}$ shrinks significantly for $M=10^{9} \mathrm{TeV}$, as can be seen in Fig. 5 and Fig. 6. This is because, the significant part of the parameter space is discarded by the stability condition at large $M$ where the neutrino Yukawa couplings becomes large $\mathcal{O}\left(10^{-1}\right)$. Such large Yukawa coupling contributes to the beta function of $\lambda_{2}$ as shown in Eq. 24b through the terms $+\lambda_{2} y_{\nu}^{2}$ and $-y_{\nu}^{4}$ that either makes $\lambda_{2}$ perturbative in some cases or $\lambda_{2}$ negative and the vacuum unstable in other. We elaborately discuss this feature below with some benchmark points.

We have selected two benchmark points(BP1 and $\mathrm{BP} 2)$ as samples out of the allowed regions consistent with the relic density constraints. These points demonstrate how the different theoretical constraints can truncate the scale of validity of this scenario for two different values of right-handed neutrino masses $M=110 \mathrm{TeV}$ and $M=10^{9} \mathrm{TeV}$ respectively. The parameter values are listed in Table. 1 . $\mathrm{BP} 1$ and BP2 yield $\Omega_{\mathrm{DM}} h^{2}=0.1151$ and 0.1207 respectively, which is within with the allowed range of relic density. For $M=110 \mathrm{TeV}, \mathrm{BP} 1$ ensures a stable vacuum till the Planck scale (Fig. 7a). It is 

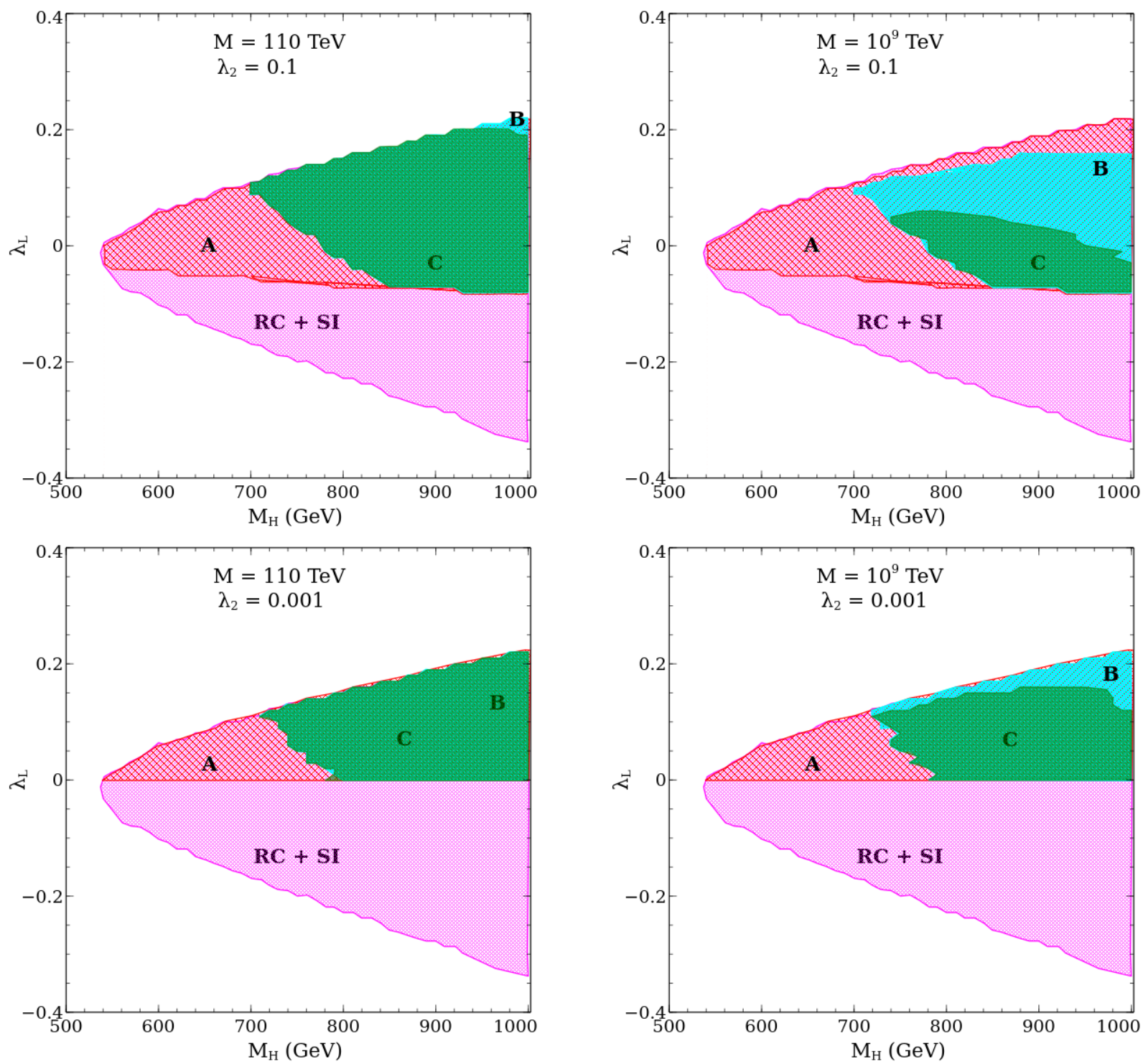

Figure 5: Region(s) allowed in the $M_{H^{-}} \lambda_{L}$ plane obeying the various constraints for $M=110 \mathrm{TeV}$ (left panel) and $M=10^{9} \mathrm{TeV}$ (right panel) with three different choices of $\Lambda_{U V}$ and two values of $\lambda_{2}$ (upper and lower panel). The full region (marked by $\mathbf{R C}+\mathbf{S I}$ ) (magenta) is allowed by the DM constraints alone. The overlapped regions labeled by $\mathbf{A}$ (red), $\mathbf{B}$ (cyan) and $\mathbf{C}$ (green) are consistent with the theoretical constraints up to $\Lambda_{U V}=10^{6}, 10^{16}$ and $10^{19} \mathrm{GeV}$, respectively. 

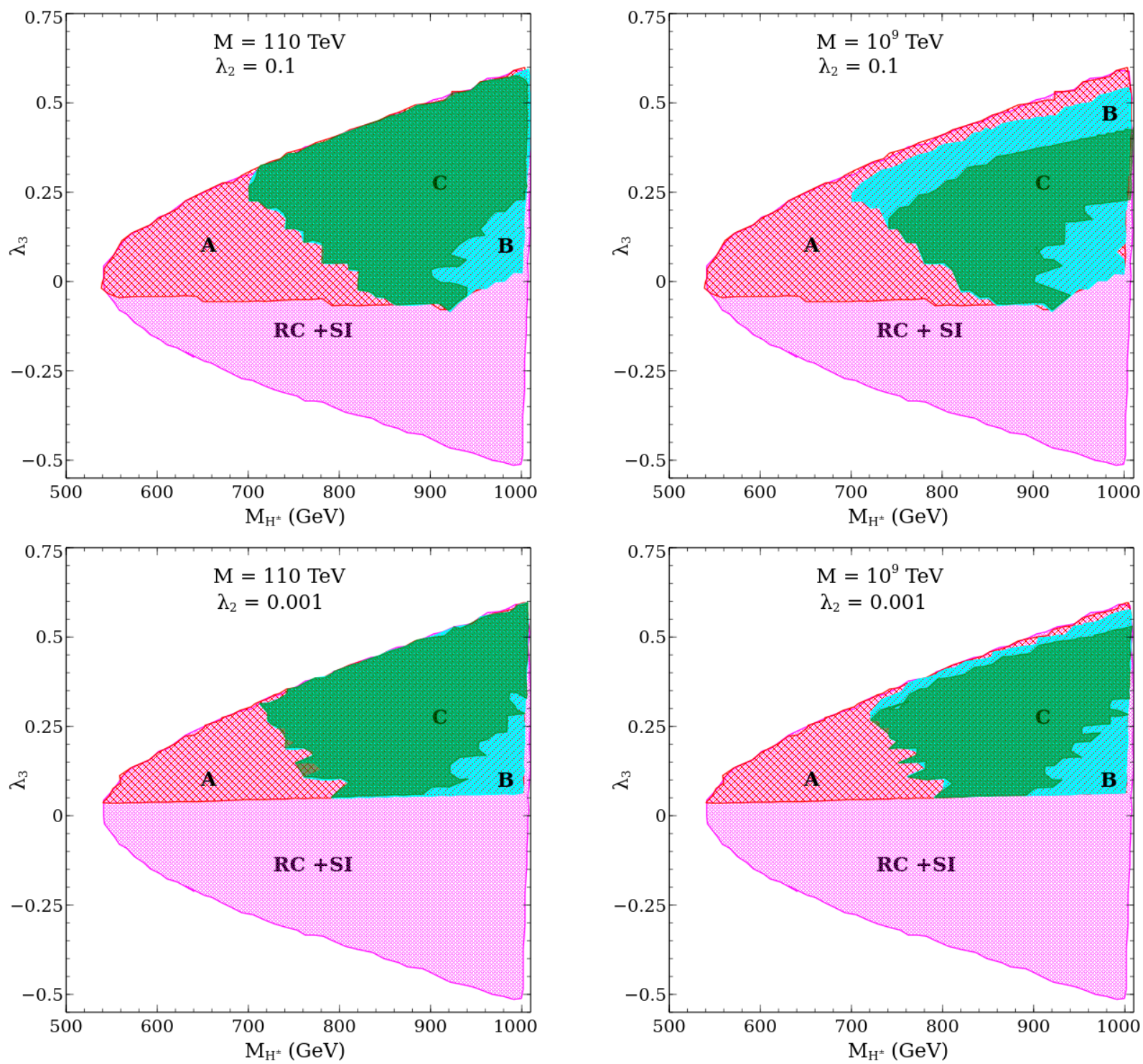

Figure 6: Region(s) allowed in the $M_{H}^{ \pm}$- $\lambda_{3}$ plane obeying the various constraints for $M=110 \mathrm{TeV}$ (left panel) and $M=10^{9} \mathrm{TeV}$ (right panel) with three different choices of $\Lambda_{U V}$ and two values of $\lambda_{2}$ (upper and lower panel). The full region (marked by $\mathbf{R C}+\mathbf{S I}$ ) (magenta) is allowed by the DM constraints alone. The overlapped regions labeled by $\mathbf{A}$ (red), $\mathbf{B}$ (cyan) and $\mathbf{C}$ (green) are consistent with the theoretical constraints up to $\Lambda_{U V}=10^{6}, 10^{16}$ and $10^{19} \mathrm{GeV}$, respectively. 


\begin{tabular}{|c|c|c|c|c|c|}
\hline BP & $M_{H}$ & $M_{H^{ \pm}}$ & $M_{A}$ & $\lambda_{L}$ & $\lambda_{2}$ \\
\hline \hline BP1 & $850.0 \mathrm{GeV}$ & $854.0 \mathrm{GeV}$ & $858.0 \mathrm{GeV}$ & 0.02 & 0.1 \\
\hline BP2 & $710.0 \mathrm{GeV}$ & $712.0 \mathrm{GeV}$ & $711.0 \mathrm{GeV}$ & 0.11 & 0.1 \\
\hline \hline
\end{tabular}

Table 1: Benchmark values (BP) of parameters affecting the RG evolution of the quartic couplings. For each $\mathrm{BP}$, two values of $M$, namely, $110 \mathrm{TeV}$ and $10^{9} \mathrm{TeV}$, have been used.

also consistent throughout with perturbativity and unitarity. However, one has $y_{\nu}=0.168$ at $M=10^{9}$ $\mathrm{TeV}$. For this value, the term $\mathcal{O}\left(\lambda_{2} y_{\nu}^{2}\right)$ has a dominant role in the RG evolution and $\lambda_{2}$ starts increasing rapidly from $10^{9} \mathrm{TeV}$ on wards (Fig. $7 \mathrm{~b}$ ).

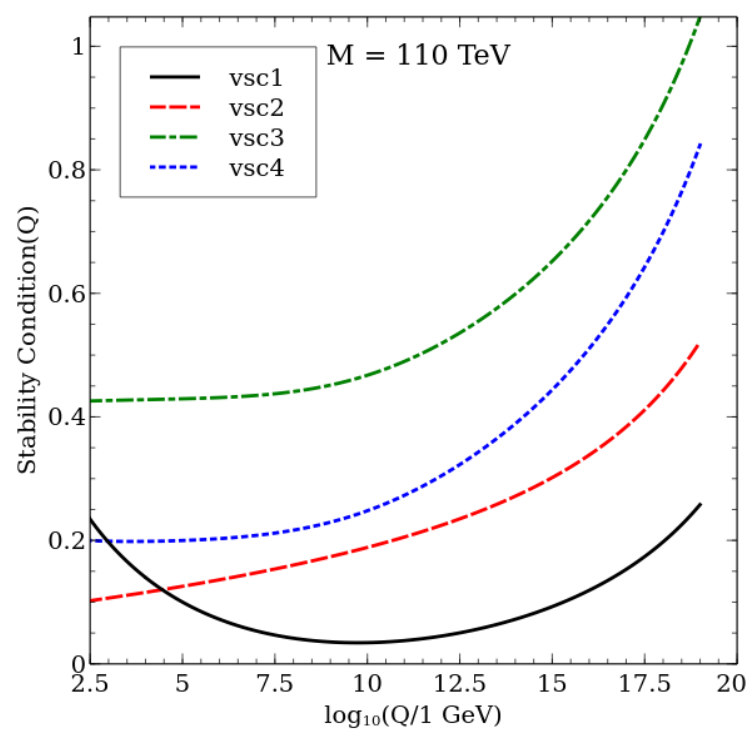

(a)

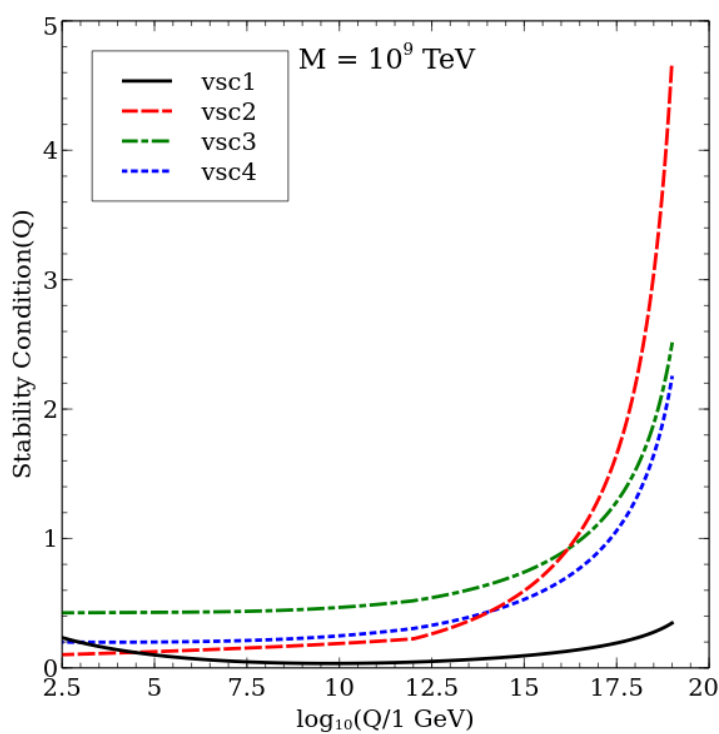

(b)

Figure 7: RG running of different scalar quartic couplings corresponding to BP1. The solid, dashed, dashed dotted and dotted lines denote the evolution curves of the stability conditions vsc1, vsc2, vsc3 and vsc4 respectively.

For $M=110 \mathrm{TeV}, \mathrm{BP} 2$ exhibits similar features in the evolution trajectory as in BP1 (Fig. 8a). For $M=10^{9} \mathrm{TeV}$, the Yukawa coupling $y_{\nu}$ starts with an initial value around 0.51 . This is accounted for by the very small mass splitting, of the order of a $\mathrm{GeV}$, between $H$ and $A$. Thus, the dominant contribution from the RH neutrinos comes from the $\mathcal{O}\left(y_{\nu}^{4}\right)$ term that causes $\lambda_{2}$ to drop sharply (Fig. 8b). Hence, in $\mathrm{BP} 2$, vacuum stability is destroyed shortly after $10^{9} \mathrm{TeV}$ as the condition $\lambda_{2}>0$ gets violated. This particular feature can only be witnessed in the case of closely spaced $M_{H}$ and $M_{A}$, which is the primary requirement to satisfy the relic density constraints discussed before. This completes the explanation of how the allowed area can shrink due to different theoretical constraints for $M=10^{9} \mathrm{TeV}$. 


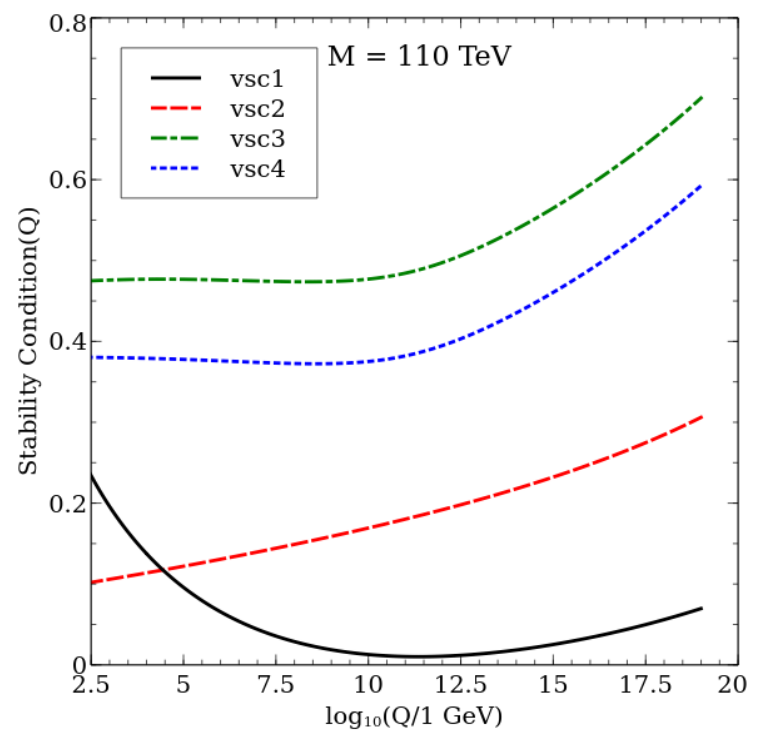

(a)

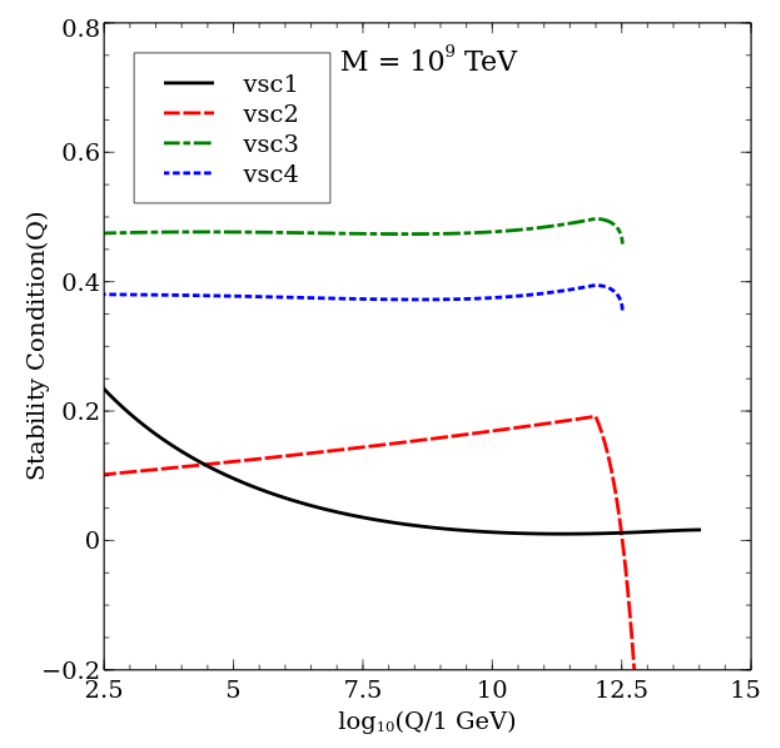

(b)

Figure 8: Same as Fig. 7 but for the benchmark point BP2.

\section{Summary and Conclusions}

We have examined the high-scale validity of a scenario that (a) offers a scalar dark matter, (b) radiatively generates Majorana masses for neutrinos, and (c) is responsible for leptogenesis. For this, we extend the SM fields with one additional inert Higgs doublet field $\left(\Phi_{2}\right)$ and three right handed neutrinos $\left(N_{i}\right)$. These new particles are odd under a discrete $Z_{2}$ symmetry, while all the SM particles are even. Because of this discrete symmetry, $\Phi_{2}$ does not acquire any vacuum expectation value (vev) and has no tree-level couplings to fermions. In this scenario, one has five physical scalars $\left(h, H, A, H^{ \pm}\right)$, where, $h$ is denoted as the SM like Higgs boson with a mass of $125 \mathrm{GeV}$. The lightest state between $H$ and $A$ is the dark matter candidate due to built in $Z_{2}$ symmetry. In our analysis we have assumed $H$ to be the dark matter candidate. Neutrino masses are generated at the one-loop level. The neutrino masses and mixing angles are determined in terms of Yukawa couplings $\left(y_{\nu}\right)$, new Higgs particle masses $\left(M_{H}, M_{A}\right)$ and three heavy Majorana masses $\left(M_{1,2,3}\right)$. In our numerical analysis we have assumed $M_{1}$ is mass of the lightest state and considered two values, namely, $M_{1} \equiv M=110 \mathrm{TeV}$ and $10^{9} \mathrm{TeV}$. These two mass scales are consistent with Leptogenesis . For simplicity, in our analysis, we have considered only one diagonal Yukawa coupling and to determine the value of this coupling we have scanned over $M_{H}$ and $M_{A}$ for a given value of $M$, by keeping $M_{\nu} \sim \mathcal{O}(0.1 \mathrm{eV})$.

The parameter space of this model is determined in terms of additional Higgs boson masses, $M_{H}, M_{A}, M_{H^{ \pm}}$, one quartic coupling $\lambda_{2}$ and a set of quartic coupling combinations, $\lambda_{L}$. While scanning the parameter space of this model, we have imposed the LEP bound on scalar masses, $M_{H}, M_{A}$ and $M_{H^{ \pm}}$.

As far as the dark matter constraints are concerned, we have used the relic density limits obtained at $3 \sigma$ uncertainty from the PLANCK experiment and the direct detection cross-section limit from the 
LUX experiment. Finally, for $M_{H}<M_{h} / 2$, which would lead to large invisible decay width of the SM like Higgs boson, we demanded that the corresponding branching ratio is less than $20 \%$ as obtained from the model independent Higgs precision analysis.

With these set of constraints in hand, we have then scanned the IDM parameter space for two different ranges of dark matter masses, $50 \mathrm{GeV}<M_{H}<90 \mathrm{GeV}$ and $M_{H}>500 \mathrm{GeV}$. It should be noted that with $M_{h}$ fixed at $125 \mathrm{GeV}, \lambda_{2}, \lambda_{L}, M_{H}, M_{A}$ and $M_{H^{ \pm}}$determined all the remaining quartic couplings. We have used these quartic couplings as the electroweak boundary condition by setting the starting RG running scale $Q=M_{t}$ and evolved these couplings up to the scale $\Lambda_{\mathrm{UV}}$, where this scenario remained consistent. In the RG evolution of these quartic couplings, the neutrino Yukawa couplings started playing its role from the right handed neutrino mass scale $Q=M$ on wards. Following are the salient features of this model that our analysis brings out.

- In our study we have explicitly demonstrated that at the low DM mass region, the vacuum stability, perturbativity and unitarity constraints put stringent limits on the low-energy value of the coupling $\lambda_{L}$ and the $Z_{2}$-odd scalar masses $M_{H^{ \pm}}, M_{A}$. These bounds strongly depend upon the scale up to which the theory is valid and the right-handed neutrino mass scale. It is interesting to note that all the parameter space allowed by the DM relic density and direct detection constraints lies well within the region allowed by the theoretical constraints valid up to the Planck scale. However, once we have imposed the condition that the Higgs to diphoton signal strength $\left(\mu_{\gamma \gamma}\right)$ should lie within $2 \sigma$ of the weighted average value of the ATLAS and CMS data, the allowed parameter space further squeezed.

- The scenario with high DM mass region $\left(M_{H}>500 \mathrm{GeV}\right)$ is significantly different from that of the low DM mass region. In this case, the DM being very heavy, the constraints from direct detection is rather insignificant. On the other hand, the relic density constraint is ensured by a degenerate $Z_{2}$-odd scalars $(\Delta M \simeq 10 \mathrm{GeV})$. As a result of these, a large part of the parameter space in $\lambda_{L}-M_{H}$ and $\lambda_{3}-M_{H^{ \pm}}$planes remain unconstrained.

- However, the study of the high scale validity of high DM mass region has interesting consequences. The DM-allowed region reduces substantially after imposing the theoretical constraints and this reduction is strongly dependent on the cut-off scale $\left(\Lambda_{\mathrm{UV}}\right)$. The effect of neutrino Yukawa couplings in the RG evolution of the different quartic couplings are also prominent in this case. We have found that with the increase in the right handed neutrino mass scale $M$, the neutrino Yukawa coupling $\left(y_{\nu}\right)$ also increases, which in turn further reduces the allowed parameter space by either destabilizing the vacuum or violating the perturbativity bound. There is nonetheless a clearly identifiable region of the parameter space, which keeps the model valid all the way up to the Planck scale. This scenario is consistent with the measured Higgs-to-diphoton rates as measured during the $8 \mathrm{TeV}$ run of the LHC.

\section{Acknowledgments :}

The work of $\mathrm{NC}$ and $\mathrm{BM}$ was partially supported by funding available from the Department of Atomic Energy, Government of India, for the Regional Centre for Accelerator-based Particle Physics (RECAPP), Harish-Chandra Research Institute. DKG and IS acknowledge the hospitality of RECAPP and High Energy Physics Division, ICTP during the early stage of this project, while NC and BM 
thank the Department of Theoretical Physics, Indian Association for the Cultivation of Science at a later stage.

\section{Appendix}

\section{A One-loop Renormalization group ( $R G)$ equations}

The RG equations for the gauge couplings, for this model, are given by [42],

$$
\begin{aligned}
16 \pi^{2} \frac{d g_{s}}{d t} & =-7 g_{s}^{3}, \\
16 \pi^{2} \frac{d g}{d t} & =-3 g^{3}, \\
16 \pi^{2} \frac{d g^{\prime}}{d t} & =7 g^{\prime 3} .
\end{aligned}
$$

Here $g^{\prime}, g$ and $g_{s}$ denote the $\mathrm{U}(1), \mathrm{SU}(2)_{L}$ and $\mathrm{SU}(3)_{c}$ gauge couplings respectively.

The quartic couplings $\lambda_{i}(i=1, \ldots, 5)$ evolve according to,

$$
\begin{aligned}
16 \pi^{2} \frac{d \lambda_{1}}{d t}= & 12 \lambda_{1}^{2}+4 \lambda_{3}^{2}+4 \lambda_{3} \lambda_{4}+2 \lambda_{4}^{2}+2 \lambda_{5}^{2}+\frac{3}{4}\left(3 g^{4}+g^{\prime 4}+2 g^{2} g^{\prime 2}\right) \\
& -\lambda_{1}\left(9 g^{2}+3 g^{\prime 2}-12 y_{t}^{2}-12 y_{b}^{2}-4 y_{\tau}^{2}\right)-12 y_{t}^{4}, \\
16 \pi^{2} \frac{d \lambda_{2}}{d t}= & 12 \lambda_{2}^{2}+4 \lambda_{3}^{2}+4 \lambda_{3} \lambda_{4}+2 \lambda_{4}^{2}+2 \lambda_{5}^{2} \\
& +\frac{3}{4}\left(3 g^{4}+g^{\prime 4}+2 g^{2} g^{\prime 2}\right)-3 \lambda_{2}\left(3 g^{2}+g^{\prime 2}-\frac{4}{3} y_{\nu}^{2}\right)-4 y_{\nu}^{4}, \\
16 \pi^{2} \frac{d \lambda_{3}}{d t}= & \left(\lambda_{1}+\lambda_{2}\right)\left(6 \lambda_{3}+2 \lambda_{4}\right)+4 \lambda_{3}^{2}+2 \lambda_{4}^{2}+2 \lambda_{5}^{2}+\frac{3}{4}\left(3 g^{4}+g^{\prime 4}-2 g^{2} g^{\prime 2}\right) \\
& -\lambda_{3}\left(9 g^{2}+3 g^{\prime 2}-6 y_{t}^{2}-6 y_{b}^{2}-2 y_{\tau}^{2}-2 y_{\nu}^{2}\right), \\
16 \pi^{2} \frac{d \lambda_{4}}{d t}= & 2\left(\lambda_{1}+\lambda_{2}\right) \lambda_{4}+8 \lambda_{3} \lambda_{4}+4 \lambda_{4}^{2}+8 \lambda_{5}^{2}+3 g^{2} g^{\prime 2} \\
& -\lambda_{4}\left(9 g^{2}+3 g^{\prime 2}-6 y_{t}^{2}-6 y_{b}^{2}-2 y_{\tau}^{2}-2 y_{\nu}^{2}\right), \\
16 \pi^{2} \frac{d \lambda_{5}}{d t}= & \left(2 \lambda_{1}+2 \lambda_{2}+8 \lambda_{3}+12 \lambda_{4}\right) \lambda_{5}-\lambda_{5}\left(9 g^{2}+3 g^{\prime 2}-6 y_{b}^{2}-2 y_{\tau}^{2}-6 y_{t}^{2}-2 y_{\nu}^{2}\right),
\end{aligned}
$$

For the Yukawa couplings the corresponding set of RG equations are,

$$
\begin{aligned}
16 \pi^{2} \frac{d y_{b}}{d t} & =y_{b}\left(-8 g_{s}^{2}-\frac{9}{4} g^{2}-\frac{5}{12} g^{\prime 2}+\frac{9}{2} y_{b}^{2}+y_{\tau}^{2}+\frac{3}{2} y_{t}^{2}\right), \\
16 \pi^{2} \frac{d y_{t}}{d t} & =y_{t}\left(-8 g_{s}^{2}-\frac{9}{4} g^{2}-\frac{17}{12} g^{\prime 2}+\frac{9}{2} y_{t}^{2}+y_{\tau}^{2}+\frac{3}{2} y_{b}^{2}\right), \\
16 \pi^{2} \frac{d y_{\tau}}{d t} & =y_{\tau}\left(-\frac{9}{4} g^{2}-\frac{15}{4} g^{\prime 2}+3 y_{b}^{2}+3 y_{t}^{2}+\frac{1}{2} y_{\nu}^{2}+\frac{5}{2} y_{\tau}^{2}\right) . \\
16 \pi^{2} \frac{d y_{\nu}}{d t} & =y_{\tau}\left(-\frac{9}{4} g^{2}-\frac{3}{4} g^{\prime 2}-\frac{3}{4} y_{\tau}^{2}+\frac{5}{2} y_{\nu}^{2}\right) .
\end{aligned}
$$




\section{References}

[1] ATLAS Collaboration, G. Aad et. al., Observation of a new particle in the search for the Standard Model Higgs boson with the ATLAS detector at the LHC, Phys.Lett. B716 (2012) 1-29, [arXiv: 1207.7214].

[2] CMS Collaboration, S. Chatrchyan et. al., Observation of a new boson at a mass of $125 \mathrm{GeV}$ with the CMS experiment at the LHC, Phys.Lett. B716 (2012) 30-61, [arXiv:1207.7235].

[3] G. Degrassi, S. Di Vita, J. Elias-Miro, J. R. Espinosa, G. F. Giudice, et. al., Higgs mass and vacuum stability in the Standard Model at NNLO, JHEP 1208 (2012) 098, [arXiv: 1205.6497].

[4] E. Ma, Verifiable radiative seesaw mechanism of neutrino mass and dark matter, Phys.Rev. D73 (2006) 077301, [hep-ph/0601225].

[5] N. G. Deshpande and E. Ma, Pattern of Symmetry Breaking with Two Higgs Doublets, Phys.Rev. D18 (1978) 2574.

[6] R. Barbieri, L. J. Hall, and V. S. Rychkov, Improved naturalness with a heavy Higgs: An Alternative road to LHC physics, Phys.Rev. D74 (2006) 015007, [hep-ph/0603188].

[7] L. Lopez Honorez, E. Nezri, J. F. Oliver, and M. H. Tytgat, The Inert Doublet Model: An Archetype for Dark Matter, JCAP 0702 (2007) 028, [hep-ph/0612275].

[8] Q.-H. Cao, E. Ma, and G. Rajasekaran, Observing the Dark Scalar Doublet and its Impact on the Standard-Model Higgs Boson at Colliders, Phys.Rev. D76 (2007) 095011, [arXiv:0708.2939].

[9] S. Andreas, M. H. Tytgat, and Q. Swillens, Neutrinos from Inert Doublet Dark Matter, JCAP 0904 (2009) 004, [arXiv:0901.1750].

[10] E. Nezri, M. H. Tytgat, and G. Vertongen, e+ and anti-p from inert doublet model dark matter, JCAP 0904 (2009) 014, [arXiv:0901.2556].

[11] T. Hambye, F.-S. Ling, L. Lopez Honorez, and J. Rocher, Scalar Multiplet Dark Matter, JHEP 0907 (2009) 090, [arXiv:0903.4010].

[12] L. Lopez Honorez and C. E. Yaguna, The inert doublet model of dark matter revisited, JHEP 1009 (2010) 046, [arXiv: 1003.3125].

[13] L. Lopez Honorez and C. E. Yaguna, A new viable region of the inert doublet model, JCAP 1101 (2011) 002, [arXiv:1011.1411].

[14] E. Dolle, X. Miao, S. Su, and B. Thomas, Dilepton Signals in the Inert Doublet Model, Phys.Rev. D81 (2010) 035003, [arXiv:0909.3094].

[15] X. Miao, S. Su, and B. Thomas, Trilepton Signals in the Inert Doublet Model, Phys.Rev. D82 (2010) 035009, [arXiv: 1005.0090].

[16] A. Arhrib, R. Benbrik, and N. Gaur, $H \rightarrow \gamma \gamma$ in Inert Higgs Doublet Model, Phys.Rev. D85 (2012) 095021, [arXiv:1201.2644]. 
[17] M. Gustafsson, S. Rydbeck, L. Lopez-Honorez, and E. Lundstrom, Status of the Inert Doublet Model and the Role of multileptons at the LHC, Phys.Rev. D86 (2012) 075019, [arXiv:1206.6316].

[18] B. Swiezewska and M. Krawczyk, Diphoton rate in the inert doublet model with a 125 GeV Higgs boson, Phys.Rev. D88 (2013), no. 3 035019, [arXiv:1212.4100].

[19] A. Goudelis, B. Herrmann, and O. StÃěl, Dark matter in the Inert Doublet Model after the discovery of a Higgs-like boson at the LHC, JHEP 1309 (2013) 106, [arXiv:1303.3010].

[20] M. Krawczyk, D. Sokolowska, and B. Swiezewska, Inert Doublet Model with a 125 GeV Higgs, arXiv:1304.7757.

[21] M. Krawczyk, D. SokoÁĆowska, P. Swaczyna, and B. ǍŽwieÅijewska, Higgs $\rightarrow \gamma \gamma, Z \gamma$ in the Inert Doublet Model, Acta Phys.Polon. B44 (2013), no. 11 2163-2170, [arXiv:1309.7880].

[22] A. Arhrib, Y.-L. S. Tsai, Q. Yuan, and T.-C. Yuan, An Updated Analysis of Inert Higgs Doublet Model in light of the Recent Results from LUX, PLANCK, AMS-02 and LHC, arXiv:1310.0358.

[23] A. Arhrib, R. Benbrik, and T.-C. Yuan, Associated Production of Higgs at Linear Collider in the Inert Higgs Doublet Model, Eur.Phys.J. C74 (2014) 2892, [arXiv:1401.6698].

[24] E. Ma, Common origin of neutrino mass, dark matter, and baryogenesis, Mod.Phys.Lett. A21 (2006) 1777-1782, [hep-ph/0605180].

[25] L. M. Krauss, S. Nasri, and M. Trodden, A Model for neutrino masses and dark matter, Phys.Rev. D67 (2003) 085002, [hep-ph/0210389].

[26] J. Kubo, E. Ma, and D. Suematsu, Cold Dark Matter, Radiative Neutrino Mass, $\mu \rightarrow e \gamma$, and Neutrinoless Double Beta Decay, Phys.Lett. B642 (2006) 18-23, [hep-ph/0604114].

[27] D. Aristizabal Sierra, J. Kubo, D. Restrepo, D. Suematsu, and O. Zapata, Radiative seesaw: Warm dark matter, collider and lepton flavour violating signals, Phys.Rev. D79 (2009) 013011, [arXiv: 0808.3340].

[28] D. Suematsu, T. Toma, and T. Yoshida, Reconciliation of CDM abundance and $\mu \rightarrow e \gamma$ in a radiative seesaw model, Phys.Rev. D79 (2009) 093004, [arXiv:0903.0287].

[29] D. Suematsu, T. Toma, and T. Yoshida, Enhancement of the annihilation of dark matter in a radiative seesaw model, Phys.Rev. D82 (2010) 013012, [arXiv:1002.3225].

[30] D. Suematsu, Thermal Leptogenesis in a TeV Scale Model for Neutrino Masses, Eur.Phys.J. C72 (2012) 1951, [arXiv:1103.0857].

[31] R. Bouchand and A. Merle, Running of Radiative Neutrino Masses: The Scotogenic Model, JHEP 1207 (2012) 084, [arXiv:1205.0008].

[32] S. Kashiwase and D. Suematsu, Baryon number asymmetry and dark matter in the neutrino mass model with an inert doublet, Phys.Rev. D86 (2012) 053001, [arXiv:1207.2594].

[33] S. Kashiwase and D. Suematsu, Leptogenesis and dark matter detection in a TeV scale neutrino mass model with inverted mass hierarchy, Eur.Phys.J. C73 (2013) 2484, [arXiv:1301.2087]. 
[34] T. Toma and A. Vicente, Lepton Flavor Violation in the Scotogenic Model, JHEP 1401 (2014) 160, [arXiv: 1312 . 2840].

[35] H. Davoudiasl and I. M. Lewis, Right-Handed Neutrinos as the Origin of the Electroweak Scale, Phys.Rev. D90 (2014), no. 3 033003, [arXiv:1404.6260].

[36] M. Fukugita and T. Yanagida, Baryogenesis Without Grand Unification, Phys.Lett. B174 (1986) 45.

[37] A. Pilaftsis, CP violation and baryogenesis due to heavy Majorana neutrinos, Phys.Rev. D56 (1997) 5431-5451, [hep-ph/9707235].

[38] J. Racker, Mass bounds for baryogenesis from particle decays and the inert doublet model, JCAP 1403 (2014) 025, [arXiv: 1308.1840].

[39] M. Sher, Electroweak Higgs Potentials and Vacuum Stability, Phys.Rept. 179 (1989) 273-418.

[40] S. Nie and M. Sher, Vacuum stability bounds in the two Higgs doublet model, Phys.Lett. B449 (1999) 89-92, [hep-ph/9811234].

[41] P. Ferreira, R. Santos, and A. Barroso, Stability of the tree-level vacuum in two Higgs doublet models against charge or CP spontaneous violation, Phys.Lett. B603 (2004) 219-229, [hep-ph/0406231].

[42] G. Branco, P. Ferreira, L. Lavoura, M. Rebelo, M. Sher, et. al., Theory and phenomenology of two-Higgs-doublet models, Phys.Rept. 516 (2012) 1-102, [arXiv:1106.0034].

[43] I. Ginzburg and I. Ivanov, Tree-level unitarity constraints in the most general 2HDM, Phys.Rev. D72 (2005) 115010, [hep-ph/0508020].

[44] B. Gorczyca and M. Krawczyk, Tree-Level Unitarity Constraints for the SM-like 2HDM, arXiv:1112.5086.

[45] G. Bhattacharyya, D. Das, P. B. Pal, and M. Rebelo, Scalar sector properties of two-Higgs-doublet models with a global U(1) symmetry, JHEP 1310 (2013) 081, [arXiv: 1308.4297].

[46] N. Chakrabarty, U. K. Dey, and B. Mukhopadhyaya, High-scale validity of a two-Higgs doublet scenario: a study including LHC data, JHEP 1412 (2014) 166, [arXiv:1407.2145].

[47] E. Lundstrom, M. Gustafsson, and J. Edsjo, The Inert Doublet Model and LEP II Limits, Phys.Rev. D79 (2009) 035013, [arXiv:0810.3924].

[48] A. Pierce and J. Thaler, Natural Dark Matter from an Unnatural Higgs Boson and New Colored Particles at the TeV Scale, JHEP 0708 (2007) 026, [hep-ph/0703056].

[49] M. A. Shifman, A. Vainshtein, M. Voloshin, and V. I. Zakharov, Low-Energy Theorems for Higgs Boson Couplings to Photons, Sov.J.Nucl.Phys. 30 (1979) 711-716.

[50] J. R. Ellis, M. K. Gaillard, and D. V. Nanopoulos, A Phenomenological Profile of the Higgs Boson, Nucl.Phys. B106 (1976) 292. 
[51] R. Cahn, M. S. Chanowitz, and N. Fleishon, Higgs Particle Production by $Z \rightarrow H \gamma$, Phys.Lett. B82 (1979) 113.

[52] L. Bergstrom and G. Hulth, Induced Higgs Couplings to Neutral Bosons in $e^{+} e^{-}$Collisions, Nucl.Phys. B259 (1985) 137.

[53] A. Djouadi, The Anatomy of electro-weak symmetry breaking. II. The Higgs bosons in the minimal supersymmetric model, Phys.Rept. 459 (2008) 1-241, [hep-ph/0503173].

[54] ATLAS Collaboration, G. Aad et. al., Measurement of Higgs boson production in the diphoton decay channel in pp collisions at center-of-mass energies of 7 and 8 TeV with the ATLAS detector, Phys.Rev. D90 (2014) 112015, [arXiv:1408.7084].

[55] CMS Collaboration, V. Khachatryan et. al., Observation of the diphoton decay of the Higgs boson and measurement of its properties, Eur.Phys.J. C74 (2014), no. 10 3076, [arXiv:1407.0558].

[56] Planck Collaboration, P. Ade et. al., Planck 2013 results. XVI. Cosmological parameters, Astron.Astrophys. 571 (2014) A16, [arXiv:1303.5076].

[57] XENON100 Collaboration, E. Aprile et. al., Dark Matter Results from 225 Live Days of XENON100 Data, Phys.Rev.Lett. 109 (2012) 181301, [arXiv:1207.5988].

[58] LUX Collaboration, D. Akerib et. al., First results from the LUX dark matter experiment at the Sanford Underground Research Facility, Phys.Rev.Lett. 112 (2014), no. 9 091303, [arXiv: 1310.8214$]$.

[59] S. Banerjee, S. Mukhopadhyay, and B. Mukhopadhyaya, New Higgs interactions and recent data from the LHC and the Tevatron, JHEP 1210 (2012) 062, [arXiv:1207.3588].

[60] K. Cheung, J. S. Lee, and P.-Y. Tseng, Higgs precision analysis updates 2014, Phys.Rev. D90 (2014), no. 9 095009, [arXiv: 1407.8236].

[61] M. Klasen, C. E. Yaguna, J. D. Ruiz-Alvarez, D. Restrepo, and O. Zapata, Scalar dark matter and fermion coannihilations in the radiative seesaw model, JCAP 1304 (2013) 044, [arXiv: 1302.5298].

[62] G. Belanger, F. Boudjema, A. Pukhov, and A. Semenov, micrOMEGAs_3:A program for calculating dark matter observables, Comput.Phys.Commun. 185 (2014) 960-985, [arXiv: 1305.0237].

[63] H.-J. He, N. Polonsky, and S.-f. Su, Extra families, Higgs spectrum and oblique corrections, Phys.Rev. D64 (2001) 053004, [hep-ph/0102144].

[64] W. Grimus, L. Lavoura, O. Ogreid, and P. Osland, A Precision constraint on multi-Higgs-doublet models, J.Phys. G35 (2008) 075001, [arXiv:0711.4022]. 